\title{
The multifractal nature of heterogeneous sums of Dirac masses
}

\author{
BY JULIEN BARRAL \\ Projet SOSSO2 - INRIA Rocquencourt, B.P. 105 \\ 78153 Le Chesnay Cedex, FRANCE \\ AND STÉPHANE SEURET \\ Laboratoire d'Analyse et de Mathématiques Appliquées \\ Université Paris 12 - UFR Sciences et Technologie \\ 61, avenue du Général de Gaulle, 94010 Créteil Cedex, FRANCE \\ (Received ; revised )
}

Abstract
This article investigates the natural problem of performing the multifractal analysis of
heterogeneous sums of Dirac masses
\[ \nu=\sum_{n \geq 0} w_{n} \delta_{x_{n}} \]

where $\left(x_{n}\right)_{n \geq 0}$ is a sequence of points in $[0,1]^{d}$ and $\left(w_{n}\right)_{n \geq 0}$ is a positive sequence of weights such that $\sum_{n \geq 0} w_{n}<\infty$. We consider the case where the points $x_{n}$ are roughly uniformly distributed in $[0,1]^{d}$, and the weights $w_{n}$ depend on a random self-similar measure $\mu$, a parameter $\rho \in(0,1]$, and a sequence of positive radii $\left(\lambda_{n}\right)_{n \geq 1}$ converging to 0 in the following way

$$
w_{n}=\lambda_{n}^{d(1-\rho)} \mu\left(B\left(x_{n}, \lambda_{n}^{\rho}\right)\right)\left|\log \lambda_{n}\right|^{-2} .
$$

The measure $\nu$ has a rich multiscale structure. The computation of its multifractal spectrum is related to heterogeneous ubiquity properties of the system $\left\{\left(x_{n}, \lambda_{n}\right)\right\}_{n}$ with respect to $\mu$.

\section{Introduction and motivations}

A large literature is dedicated to the multifractal analysis of continuous singular measures possessing scaling invariance properties (see $[\mathbf{1 3}, \mathbf{3 0}, \mathbf{3 2}, \mathbf{2 9}, \mathbf{7}]$ and references therein), while a few papers investigate the multifractal nature of another very natural class of singular measures: the infinite sums of Dirac masses.

In this paper we perform the multifractal analysis of a large class of infinite sums of Dirac masses. These Dirac masses are located at roughly uniformly distributed points in a compact subset of $\mathbb{R}^{d}$, and they are weighted using a statistically self-similar multifractal measure. The study of these measures is closely related to the new results on heterogeneous ubiquity established in [11].

We start by describing a class of "homogeneous" sums of Dirac masses whose multifractal analysis is performed in $[\mathbf{1 6}]$. 
Let $\Omega$ be a compact subset of $\mathbb{R}^{d}$ such that $\operatorname{dim} \Omega>0$ (dim stands for the Hausdorff dimension) and $\|\cdot\|$ a norm on $\mathbb{R}^{d}$. Let $\left(x_{n}\right)_{n \in \mathbb{N}}$ be a sequence in $\Omega^{\mathbb{N}}$ and $\left(\lambda_{n}\right)_{n \in \mathbb{N}}$ a non-increasing sequence of positive real numbers converging to 0 . For $x \in \Omega$, the approximation degree of $x$ by the family $\left(x_{n}\right)_{n}$ relative to $\left(\lambda_{n}\right)_{n}$ is

$$
\operatorname{deg}(x)=\infty \text { if } x \in\left\{x_{n}\right\} \text { and } \operatorname{deg}(x)=\limsup _{n \rightarrow \infty} \frac{\log \left\|x-x_{n}\right\|}{\log \lambda_{n}} \text { if } x \notin\left\{x_{n}\right\},
$$

see [16]. The level sets of the function $\operatorname{deg}(\cdot)$ are then defined by $F_{\xi}=\{x: \operatorname{deg}(x)=\xi\}$ for $\xi \in(0,+\infty]$. A natural assumption is $\inf _{x \in \Omega} \operatorname{deg}(x)>0$, which can be easily normalized to have $\operatorname{deg}(x) \geq 1$ for all $x \in \Omega$. This amounts to saying that $\Omega=\bigcup_{\xi \geq 1} F_{\xi}$, which arises under the assumption

$$
\limsup _{n \rightarrow \infty} B\left(x_{n}, \lambda_{n}\right)=\bigcap_{N \geq 0} \bigcup_{n \geq N} B\left(x_{n}, \lambda_{n}\right)=\Omega
$$

For $\alpha \geq \operatorname{dim} \Omega$, consider the measure $\nu$ defined by

$$
\nu=\sum_{n \geq 0} w_{n} \delta_{x_{n}} \text { with } w_{n}=\left\{\begin{array}{ll}
\left|\log \lambda_{n}\right|^{-2} \lambda_{n}^{\alpha} & \text { if } \alpha=\operatorname{dim} \Omega \\
\lambda_{n}^{\alpha} & \text { otherwise }
\end{array},\right.
$$

where $\delta_{x}$ stands for the Dirac mass located at $x \in \mathbb{R}^{d}$.

Suppose that the system $\left\{\left(x_{n}, \lambda_{n}\right)\right\}_{n \in \mathbb{N}}$ is "sparse" in the sense of [16], i.e.

$$
\exists C^{\prime}>0, \forall x \in \Omega, \forall j \in \mathbb{N}, \#\left\{n: 2^{-j} \leq \lambda_{n}<2^{-j+1}, x_{n} \in B\left(x, 2^{-j}\right)\right\} \leq C^{\prime} .
$$

Suppose also that $\Omega$ can be endowed with a monofractal finite Borel measure $m$, i.e. there exist $r_{0}>0$ and a constant $C>0$ such that $\forall x \in \Omega$ and $\forall 0<r \leq r_{0}$, we have $C^{-1} r^{\operatorname{dim} \Omega} \leq m(B(x, r)) \leq C r^{\operatorname{dim} \Omega}$.

Then the measure $\nu$ is finite and its Hausdorff multifractal spectrum is found in [16]. Recall that this spectrum is defined as follows. If $\mu$ is a positive Borel measure on $\Omega$ then the lower Hölder exponent of $\mu$ at $x$ is

$$
h_{\mu}(x)=\liminf _{r \rightarrow 0^{+}} \frac{\log \mu(B(x, r))}{\log r},
$$

and the level sets of the lower Hölder exponent function are defined as

$$
E_{h}^{\mu}=\left\{x \in \Omega: h_{\mu}(x)=h\right\} \quad(h \geq 0) .
$$

The Hausdorff multifractal spectrum of $\mu$ is the mapping $d_{\mu}: h \geq 0 \mapsto \operatorname{dim} E_{\mu}^{h}$.

For the measure $\nu$ defined above on $\Omega$, if both (1.1) and (1.3) hold true, then it is proved in [16] that the lower Hölder exponent of $\nu$ is directly deduced from $\operatorname{deg}(x)$ by the relation $h_{\nu}(x)=\alpha(\operatorname{deg}(x))^{-1}$ (in fact, only in the case $\alpha=\operatorname{dim} \Omega$ is treated, nevertheless the case $\alpha>\operatorname{dim} \Omega$ is similar). Then the Hausdorff dimension of the sets $E_{\nu}^{h}$ is closely related to the Hausdorff dimension of the sets $F_{\xi}$. The main point is that the Hausdorff dimensions of $F_{\xi}$ can be computed in the context where $\Omega$ can be endowed by a monofractal measure $m$, using results on ubiquitous systems [14]. The following theorem is obtained in [16] (Corollary 5):

Theorem $1 \cdot 1$.

(i) For every $x \in \Omega, h_{\nu}(x)=\alpha(\operatorname{deg}(x))^{-1}$. Equivalently, for every $\xi \in[1, \infty]$, $E_{\alpha / \xi}^{\nu}=F_{\xi}$. 
(ii) For every $h \in[0, \alpha], d_{\nu}(h)=(\operatorname{dim} \Omega) h / \alpha$.

A similar result holds for Lévy subordinator $[\mathbf{1 2}, \mathbf{2 0}]$. Indeed, the derivative of such a subordinator takes the same form as $(1 \cdot 2)$ when restricted to any non-trivial compact subinterval of $\mathbb{R}_{+}$. Comparable multifractal properties are also obtained for sums of Dirac masses located on dyadic points of $[0,1]$ in $[\mathbf{2}, \mathbf{8}]$, and for functions with countable and dense set of jump points like the Riemann function and general Lévy processes $[\mathbf{1 9}, \mathbf{2 0}]$.

We observe that for each $n$, the weight $w_{n}$ of the Dirac mass at $x_{n}$ does not depend on the location (in space) of $x_{n}$, but on $\lambda_{n}$ only. Consequently, using (1.1) and (1.3), the weights $w_{n}$ are roughly homogeneously distributed. This plays a central role in Theorem 1.1. This also raises the much more general problem of performing the multifractal analysis of sums of Dirac masses heterogeneously weighted

$$
\nu=\sum_{n \in \mathbb{N}} w\left(x_{n}, \lambda_{n}\right) \delta_{x_{n}},
$$

as well as finding the counterpart of the ubiquity properties used in the analysis above to this heterogeneous case.

In this paper, we resolve this problem when the heterogeneity in the weight's distribution is governed by a (possibly) multifractal measure. The set $\Omega$ is $[0,1]^{d}$. We consider a positive and finite Borel measure $\mu$ such that $\operatorname{supp}(\mu)=\Omega$ and a parameter $\rho \in(0,1]$. A property slightly weaker than (1.3) is assumed:

Definition 1.2. For every $j \geq 0$, define

$$
T_{j}=\left\{n: 2^{-(j+1)}<\lambda_{n} \leq 2^{-j}\right\} .
$$

The system $\left\{\left(x_{n}, \lambda_{n}\right)\right\}_{n \in \mathbb{N}}$ is said to be weakly redundant when there exists a sequence of integers $\left(N_{j}\right)_{j \geq 0}$ such that

(i) $\lim _{j \rightarrow \infty} \frac{\log _{2} N_{j}}{j}=0$.

(ii) for every $j \geq 1, T_{j}$ can be decomposed into $N_{j}$ pairwise disjoint subsets (denoted $\left.T_{j, 1}, \ldots, T_{j, N_{j}}\right)$ such that for each $1 \leq i \leq N_{j}$, the family $\left\{B\left(x_{n}, \lambda_{n}\right): n \in T_{j, i}\right\}$ is composed of disjoint balls. (By convention, $N_{j}=1$ if $T_{j}=\emptyset$.)

When the system $\left\{\left(x_{n}, \lambda_{n}\right)\right\}_{n \in \mathbb{N}}$ is weakly redundant, we shall study the multifractal nature of the finite Borel measure $\nu_{\rho}(0<\rho \leq 1)$ defined as

$$
\nu_{\rho}=\sum_{n \in \mathbb{N}} a_{n} \lambda_{n}^{d(1-\rho)} \mu\left(B\left(x_{n}, \lambda_{n}^{\rho}\right)\right) \delta_{x_{n}},
$$

where $a_{n}=\left|\log \lambda_{n}\right|^{-2} c_{n}^{-1}$ and $c_{n}=N_{j}$ if $n \in T_{j}$. The term $a_{n}$ is a natural normalization factor that satisfies $\left|\log a_{n}\right|=o\left(\left|\log \lambda_{n}\right|\right)$ and makes the measure $\nu_{\rho}$ finite.

It is easily observed that up to a multiplicative constant, if $\mu$ equals the $d$-dimensional Lebesgue measure $\ell$, then for every $\rho \in(0,1]$ the measure $\nu_{\rho}$ coincides with the measure considered in [16], i.e. the measure (1.2) when $\Omega=[0,1]^{d}$ and $\alpha=d$. Formula (1.6) is thus a natural "heterogeneous" extension of the previous "homogeneously distributed" measures. We shall emphasize the important role played by the dilation parameter $\rho$ : the multifractal behavior of $\nu_{\rho}$ when $\rho<1$ strongly differs from the behavior of $\nu_{1}$. We also mention that a preliminary result is obtained in $[8]$ in the special case when $\rho=1$, $d=1$, and the system $\left\{\left(x_{n}, \lambda_{n}\right)\right\}_{n}$ is equal to $\left\{\left(k b^{-j}, 2^{-j}\right)\right\}_{j \geq 0, k \in\left\{0, \ldots, 2^{j}-1\right\}}$. There, the hierarchical structure of the dyadic numbers considerably simplifies the discussion with respect to the much more general situation considered in this paper. 
In order to get a foretaste of our main result Theorem 2.9 , we first state one of its corollaries. Let $\mu_{0}$ be a binomial measure with weights $p_{0}>0, p_{1}>0, p_{0}+p_{1}=1$. This simple measure $\mu_{0}$ fulfills the assumptions of Theorem $2 \cdot 9$.

THEOREM 1.3. Let $\Omega=[0,1]$. Let $\left\{\left(x_{n}, \lambda_{n}\right)\right\}_{n}$ be a weakly redundant system satisfying

$$
\Omega \subset \limsup _{n \rightarrow+\infty} B\left(x_{n}, \lambda_{n} / 2\right) .
$$

Consider the measure $\nu_{1}(1 \cdot 6)$ constructed using the system $\left\{\left(x_{n}, \lambda_{n}\right)\right\}_{n}$ and $\mu_{0}$.

Let $\tau(q)=-\log _{2}\left(p_{0}^{q}+p_{1}^{q}\right)$ for $q \in \mathbb{R}$ and $\tau^{*}(h)=\inf _{q \in \mathbb{R}}(h q-\tau(q))$ for $h \geq 0$.

(i) If $0 \leq h \leq \tau^{\prime}(1)$, then $d_{\nu_{1}}(h)=h$.

(ii) Let $h \geq \tau^{\prime}(1)$. If $\tau^{*}(h)>0$, then $d_{\nu_{1}}(h)=d_{\mu_{0}}(h)=\tau^{*}(h)$, and if $\tau^{*}(h)<0$ then $E_{h}^{\nu_{1}}=\emptyset$.

We remark that $(1 \cdot 7)$ requires that $\Omega$ is covered by the balls of radii $\lambda_{n} / 2$ instead of $\lambda_{n}$ in $(1 \cdot 1)$. This slight modification is purely technical: it is due to the replacement of the Lebesgue measure by $\mu_{0}$ in $(1 \cdot 6)$ with respect to the situation described by Theorem $1 \cdot 1$.

This rather simple case of a binomial measure illustrates a phenomenon that occurs throughout the paper. The local regularity of $\nu_{\rho}$ at each point $x$ is ruled simultaneously by the behavior of the measure $\mu$ around $x$ and by the approximation degree $\operatorname{deg}(x)$ (this differs from what happens for the measure $\nu$ considered in $(1 \cdot 2)$, for which the regularity at $x$ depends only on $\operatorname{deg}(x)$ ). This combination has a repercussion on the shape of $d_{\nu_{1}}$. More specifically, the linear part in the multifractal spectrum is due to a subtle combination studied in [11] between ubiquity properties like (1.1) and the monodimensionality and self-similarity properties of the measure $\mu_{0}$.

Before making other comments, we state precisely our main result Theorem $2 \cdot 9$. This requires some definitions and technical conditions explained in Sections $2 \cdot 1$ to $2 \cdot 3$. These conditions are satisfied by large classes of measures $\mu$ possessing some statistical selfsimilarity and by many systems $\left\{\left(x_{n}, \lambda_{n}\right)\right\}_{n}$ - see Section 5 for more details - .

Subsequently we state Theorem 2.9 in Section 2.4 and we prove it in Sections 3 and 4. Theorem 2.9 extends Theorem $1 \cdot 1$, and asserts that the multifractal spectrum $d_{\nu_{\rho}}$ is composed of a linear part and a concave part, under suitable assumptions on $\mu$ and on the system $\left\{\left(x_{n}, \lambda_{n}\right)\right\}_{n}$. Consequently, the same phenomenon as the one described above for $\nu_{1}$ by Theorem 1.3 happens for more general classes of measures $\nu_{\rho}$.

\section{Statement of the main result}

In the sequel, $d \geq 1$ is fixed and $\Omega=[0,1]^{d}$. We consider a positive Borel measure $\mu$ with $\operatorname{supp}(\mu)=\Omega$, a sequence $\left(x_{n}\right)_{n} \in \Omega^{\mathbb{N}}$, a non-increasing sequence $\left(\lambda_{n}\right)_{n}$ of positive real numbers converging to zero and a parameter $0<\rho \leq 1$.

It is convenient to endow $\mathbb{R}^{d}$ with the supremum norm $\|\cdot\|_{\infty}$ and with the associated distance $(x, y) \in \mathbb{R}^{d} \times \mathbb{R}^{d} \mapsto\|x-y\|_{\infty}=\max _{1 \leq i \leq d}\left(\left|x_{i}-y_{i}\right|\right)$.

\section{$2 \cdot 1$. Some notations and definitions}

Let $c$ be an integer $\geq 2$. For every $j \geq 0$, for every $\mathbf{k}=\left(k_{1}, \ldots, k_{d}\right) \in\left\{0,1, \ldots, c^{j}-1\right\}^{d}$, $I_{j, \mathbf{k}}^{c}$ denotes the $c$-adic box $\left[k_{1} c^{-j},\left(k_{1}+1\right) c^{-j}\right) \times \ldots \times\left[k_{d} c^{-j},\left(k_{d}+1\right) c^{-j}\right)$. For every $x \in[0,1)^{d}, I_{j}^{c}(x)$ stands for the unique $c$-adic box of scale $j$ that contains $x$, and $\mathbf{k}_{j, x}^{c}$ is the unique (multi-)integer such that $I_{j}^{c}(x)=I_{j, \mathbf{k}_{j, x}^{c}}^{c}$. If $\mathbf{k}=\left(k_{1}, \ldots, k_{d}\right)$ and $\mathbf{k}^{\prime}=\left(k_{1}^{\prime}, \ldots, k_{d}^{\prime}\right)$ both belong to $\mathbb{N}^{d},\left\|\mathbf{k}-\mathbf{k}^{\prime}\right\|_{\infty}=\max _{i}\left|k_{i}-k_{i}^{\prime}\right|$. 
Throughout the paper, if $E \in \mathbb{R}^{d},|E|$ denotes the diameter of the set $E$, and if $x \in \mathbb{R}$, $[x]$ stands for the integer part of $x$.

Let $m$ be a positive Borel measure on $\mathbb{R}^{d}$ such that $\operatorname{supp}(m)=\Omega$. Let $\psi: \mathbb{R}_{+} \rightarrow \mathbb{R}_{+}$ be a non-decreasing continuous function such that $\psi(0)=0$. For every $\alpha>0$ and every subset $B$ of $\Omega$, the property $\mathcal{Q}_{\psi}^{m, \alpha}(B)$ is said to hold if

$$
|B|^{\alpha+\psi(|B|))} \leq m(B) \leq|B|^{\alpha-\psi(|B|)} .
$$

Heuristically, $\mathcal{Q}_{\psi}^{m, \alpha}(B)$ holds when $m(B) \sim|B|^{\alpha}$. For $\gamma>0, \alpha>0, \xi \geq 1$ and $0<\rho \leq 1$, we define for every $n \in \mathbb{N}$ the balls

$$
\begin{aligned}
B_{n}^{\gamma} & =B\left(x_{n}, \lambda_{n}^{\gamma}\right) \\
\text { and } \quad B_{n, \xi}(m, \rho, \alpha, \psi) & = \begin{cases}B_{n}^{\xi} & \text { if } \mathcal{Q}_{\psi}^{m, \alpha}\left(B_{n}^{\rho}\right) \text { holds } \\
\emptyset & \text { otherwise. }\end{cases}
\end{aligned}
$$

Essentially $B_{n, \xi}(m, \rho, \alpha, \psi)$ is non-empty and equal to a contracted ball $B_{n}^{\xi}$ as soon as a condition $(2 \cdot 1)$ on the larger ball $B_{n}^{\rho}$ holds.

For every $x \in \Omega$ and $\mathbf{k} \in\{-1,0,1\}^{d}$ the lower and upper Hölder exponents of a positive Borel measure $m$ at neighborhood $\mathbf{k}$ of $x$ are respectively defined by

$$
\underline{\alpha}_{m}^{\mathbf{k}}(x)=\liminf _{j \rightarrow+\infty} \frac{\log _{c} m\left(I_{j, \mathbf{k}_{j, x}^{c}+\mathbf{k}}(x)\right)}{j} \text { and } \bar{\alpha}_{m}^{\mathbf{k}}(x)=\limsup _{j \rightarrow+\infty} \frac{\log _{c} m\left(I_{j, \mathbf{k}_{j, x}^{c}+\mathbf{k}}(x)\right)}{j} .
$$

When $\underline{\alpha}_{m}^{\mathbf{k}}(x)=\bar{\alpha}_{m}^{\mathbf{k}}(x)$, their common value is denoted by $\alpha_{m}^{\mathbf{k}}(x)$ and called the Hölder exponent at neighborhood $\mathbf{k}$ of $x$. Let

$$
\widetilde{E}_{\alpha}^{m}=\left\{x \in \Omega: \forall \mathbf{k} \in\{-1,0,1\}^{d}, \alpha_{m}^{\mathbf{k}}(x)=\alpha\right\} .
$$

For $x \in \Omega$, we also need the notion of upper Hölder exponent (the counterpart of the exponent $h_{m}$ defined in (1.4))

$$
\bar{h}_{m}(x)=\limsup _{r \rightarrow 0+} \frac{\log m(B(x, r))}{\log |B(x, r)|}=\limsup _{j \rightarrow+\infty} \frac{\log m\left(B\left(x, c^{-j}\right)\right)}{\log \left|B\left(x, c^{-j}\right)\right|} .
$$

The reader can easily check that

$$
h_{m}(x)=\min _{\mathbf{k} \in\{-1,0,1\}^{d}} \underline{\alpha}_{m}^{\mathbf{k}}(x) \text { and } \bar{h}_{m}(x) \leq \min _{\mathbf{k} \in\{-1,0,1\}^{d}} \bar{\alpha}_{m}^{\mathbf{k}}(x) .
$$

The scaling function, or $L^{q}$-spectrum, associated with a measure $m$ is needed to invoke the multifractal formalism developed in [13]. For every integer $c \geq 2$, this function is defined by

$$
\tau_{m, c}: q \mapsto \lim \inf _{j \rightarrow \infty}-\frac{1}{j} \log _{c}\left(\sum_{\mathbf{k} \in\left\{0, \ldots, c^{j}-1\right\}^{d}} m\left(I_{j, \mathbf{k}}^{c}\right)^{q}\right) .
$$

Since $\operatorname{supp}(m)=\Omega=[0,1]^{d}, \tau_{m, c}$ does not depend on the integer $c \geq 2$, and is consequently denoted by $\tau_{m}$. It is a concave and non-decreasing mapping.

The Legendre transform of $\tau_{m}$ at $\alpha \in \mathbb{R}_{+}$, denoted by $\tau_{m}^{*}$, is defined by

$$
\tau_{m}^{*}: \alpha \mapsto \inf _{q \in \mathbb{R}}\left(\alpha q-\tau_{m}(q)\right) \in \mathbb{R} \cup\{-\infty\} .
$$


2.2. Irreducible elements of $\left\{x_{n}: n \geq 0\right\}$

Definition 2.1. Let $S=\left\{x_{n}: n \geq 0\right\}$ and $y \in S$. Let also $n_{y}=\min \left\{n: y=x_{n}\right\}$. The point $x_{n_{y}}$ is called the irreducible form of $y$. If $p \geq 0$ is such that $n_{x_{p}}=p, x_{p}$ is said to be irreducible.

Notice that since $\left(\lambda_{n}\right)_{n \geq 1}$ is non-increasing, $\lambda_{n_{y}} \geq \lambda_{n}$ for all $n$ such that $x_{n}=y$. This notion of irreducibility coincides with the usual notion of irreducibility when, in dimension $1,\left\{\left(x_{n}, \lambda_{n}\right)\right\}_{n}$ is a sequence taking values in the set of rational pairs $\left\{\left(p / q, 1 / q^{2}\right): q \geq\right.$ $1,0 \leq p \leq q\}$.

¿From now on, for $x \in \Omega$, we simply write $\xi_{x}$ instead of $\operatorname{deg}(x)$. The proof of the following proposition is immediate and left to the reader.

Proposition 2.2. For every $x \in \Omega$ which satisfies $\xi_{x}<\infty$, (remember that we have set $\left.\xi_{x}=\operatorname{deg}(x)\right)$

$$
\xi_{x}=\lim _{n \rightarrow \infty} \sup _{y \in S, n_{y} \geq n} \frac{\log \|x-y\|_{\infty}}{\log \lambda_{n_{y}}} .
$$

Notice that if the $x_{n}$ are pairwise distinct they are all irreducible.

2.3. Properties satisfied by the measure $\mu$ and the system $\left\{\left(x_{n}, \lambda_{n}\right)\right\}_{n}$

Definition 2.3. Property $\mathbf{P 1}(\mu)$ : There exists a constant $M$ such that

$$
\forall x \in \Omega, \forall r>0, \mu(B(x, r)) \geq r^{M} .
$$

The role of property $\mathbf{P 2}\left(\mu, \rho,\left\{\left(x_{n}, \lambda_{n}\right)\right\}, h\right)$ is to enable us to get a lower bound for the Hausdorff dimension of sets of the form $\limsup _{n \rightarrow \infty} B_{n, \xi_{n}}(\mu, \rho, h, \psi)$, where $h>0$ is a positive exponent, $\left(\xi_{n}\right)_{n \geq 0} \in[1, \infty)^{\mathbb{N}}$, and where $\psi: \mathbb{R}_{+} \rightarrow \mathbb{R}_{+}$is a non-decreasing continuous function such that $\psi(0)=0$.

Definition 2.4. Property P2 $\left(\mu, \rho,\left\{\left(x_{n}, \lambda_{n}\right)\right\}, h\right)$ and heterogeneous ubiquity: There exists a non-decreasing continuous function $\psi: \mathbb{R}_{+} \rightarrow \mathbb{R}_{+}$such that $\psi(0)=0$ and for every $\xi \geq 1$, there are a non-decreasing sequence $\left(\xi_{n}\right)_{n \geq 0}$ converging to $\xi$ and a positive Borel measure $m_{\rho, \xi}$ with the following properties: Let

$$
T=\limsup _{n \rightarrow \infty} B_{n, \xi_{n}}(\mu, \rho, h, \psi) \text { and } d\left(\tau_{\mu}^{*}(h), \rho, \xi\right)=\min \left(\frac{d(1-\rho)+\rho \tau_{\mu}^{*}(h)}{\xi}, \tau_{\mu}^{*}(h)\right)
$$

(the balls $B_{n, \xi_{n}}(\mu, \rho, h, \psi)$ are defined in (2.3)). The set $T$ is of positive $m_{\rho, \xi}$-measure (i.e. $\left.m_{\rho, \xi}(T)>0\right)$, and for every Borel set E satisfying $\operatorname{dim} E<d\left(\tau_{\mu}^{*}(h), \rho, \xi\right), m_{\rho, \xi}(E)=0$.

In particular, $\operatorname{dim} T \geq d\left(\tau_{\mu}^{*}(h), \rho, \xi\right)$.

Heuristically, $T$ is a subset of $\Omega$ which contains points which are approximated at rate $\xi \geq 1$ by some points $x_{n}$ (relatively to $\lambda_{n}$ ), those points $x_{n}$ being selected according to the $\mu$-measure of the ball $B_{n}^{\rho}=B\left(x_{n}, \lambda_{n}^{\rho}\right)$.

Property $\mathbf{P 2}\left(\mu, \rho,\left\{\left(x_{n}, \lambda_{n}\right)\right\}, h\right)$ is shown to be satisfied for large classes of systems and statistically self-similar measures in [11] (see also Section 5). It is also shown in [11] that if $\left\{\left(x_{n}, \lambda_{n}\right)\right\}_{n}$ is weakly redundant, then $\operatorname{dim} T \leq d\left(\tau_{\mu}^{*}(h), \rho, \xi\right)$.

We remark that, under the weak redundancy assumption, a saturation phenomenon occurs when $\rho<1$, in the following sense. As long as $\xi \leq \xi_{c}=\frac{d(1-\rho)+\rho \tau_{\mu}^{*}(h)}{\tau_{\mu}^{*}(h)}$, the dimension of $T$ is $\operatorname{dim} T=\tau_{\mu}^{*}(h)$, while when $\xi \geq \xi_{c}$, the dimension of $T$ starts to decrease 
The multifractal nature of heterogeneous sums of Dirac masses

and $\operatorname{dim} T=\frac{d(1-\rho)+\rho \tau_{\mu}^{*}(h)}{\xi}$. This fact plays a fundamental role in the computation of the multifractal spectrum of $\nu_{\rho}$ when $\rho<1$ in Theorem 2.9.

Definition 2.5. Property P3 $(\mu, h)$ : There exists a positive Borel measure $\mu_{h}$ with $\operatorname{supp}(\mu)=\Omega, \mu_{h}\left(\widetilde{E}_{h}^{\mu}\right)>0$, and $\mu_{h}(E)=0$ for every Borel set $E$ such that $\operatorname{dim} E<\tau_{\mu}^{*}(h)$ (the level set $\widetilde{E}_{h}^{\mu}$ is defined by (2.4)).

Property P3 $(\mu, h)$ implies the validity of the multifractal formalism for $\mu$ at $h$ in the sense that in this case $\operatorname{dim} E_{\mu}^{h}=\tau_{\mu}^{*}(h)$. In fact the inequality $\operatorname{dim} E_{\mu}^{h} \leq \tau_{\mu}^{*}(h)$ always holds true by Proposition $4 \cdot 4$.

\subsection{Statement of the main result}

Let $\mu$ be a Borel measure on $\mathbb{R}^{d}$ such that $\operatorname{supp}(\mu)=\Omega$ and a weakly redundant system $\left\{\left(x_{n}, \lambda_{n}\right)\right\}_{n}$. Let $\rho \in(0,1]$ be the dilation parameter introduced in the beginning of Section 1 in order to defined the measure $\nu_{\rho}$ in (1.6).

Two final properties are needed.

Definition 2.6. Property P4: The system $\left\{\left(x_{n}, \lambda_{n}\right)\right\}_{n}$ satisfies

$$
\Omega \backslash\left\{x_{n}: n \geq 0\right\} \subset \limsup _{n \rightarrow \infty} B\left(x_{n}, \lambda_{n} / 2\right) .
$$

As in (1.1), (2.8) ensures that $\operatorname{deg}(x)=\xi_{x} \geq 1$ for all $x \in \Omega \backslash\left\{x_{n}: n \geq 0\right\}$.

REMARK 2.7. It is important to notice that by construction (2.8) also holds if the family $\left\{\left(x_{n}, \lambda_{n}\right)\right\}_{n}$ is restricted to the pairs such that $x_{n}$ is irreducible.

Definition 2.8. Property P5 $(\mu)$ : Let $q_{c}(\mu)$ be the (critical) real number defined by

$$
q_{c}(\mu)=\inf \left\{q: \tau_{\mu}(q)=0\right\} .
$$

Due to the definition of $\tau_{\mu}(2 \cdot 5), q_{c}(\mu)$ always belongs to $(0,1]$. We assume that

$$
q_{c}(\mu)=1 .
$$

$\mathbf{P 5}(\mu)$ is satisfied for example as soon as $\mu$ is an atomless measure such that $\tau_{\mu}^{*}$ is negative in a neighborhood of $0^{+}$. This implies that the lower Hölder exponents of $\mu$ range in an interval isolated from 0 . This situation occurs for many classes of measures obtained by using a multiplicative scheme (see again Section 5 for examples). We then define

$$
h_{c}(\mu)=\tau_{\mu}^{\prime}\left(q_{c}(\mu)^{-}\right)=\tau_{\mu}^{\prime}\left(1^{-}\right) .
$$

We emphasize that in the next result, $\mathbf{P} \mathbf{1}(\mu)$ allows us to obtain an upper bound for the multifractal spectrum $d_{\nu_{\rho}}$ of $\nu_{\rho}$, and that $\mathbf{P 2}\left(\mu, \rho,\left\{\left(x_{n}, \lambda_{n}\right)\right\}, h\right)$ and $\mathbf{P} \mathbf{3}(\mu, h)$ are necessary to be able to get a lower bound for $d_{\nu_{\rho}}$.

Theorem 2.9. Let $\mu$ be a positive Borel measure on $\Omega=[0,1]^{d}$, and let $\left\{\left(x_{n}, \lambda_{n}\right)\right\}_{n}$ be a weakly redundant system in $\Omega$. Let $\rho \in(0,1]$, and $\nu_{\rho}$ be the measure obtained in (1.6). Assume that $\mathbf{P 1}(\mu), \mathbf{P} 4$ and $\mathbf{P 5}(\mu)$ together hold.

Case $\rho=1$.

(i) If $h_{c}(\mu)>0$, then $d_{\nu_{1}}(h) \leq h$ for every $h \in\left[0, h_{c}(\mu)\right]$.

Moreover, if $\mathbf{P 2}\left(\mu, 1,\left\{\left(x_{n}, \lambda_{n}\right)\right\}, h_{c}(\mu)\right)$ holds, then $d_{\nu_{1}}(h)=h$ for every $h \in$ $\left[0, h_{c}(\mu)\right]$. 


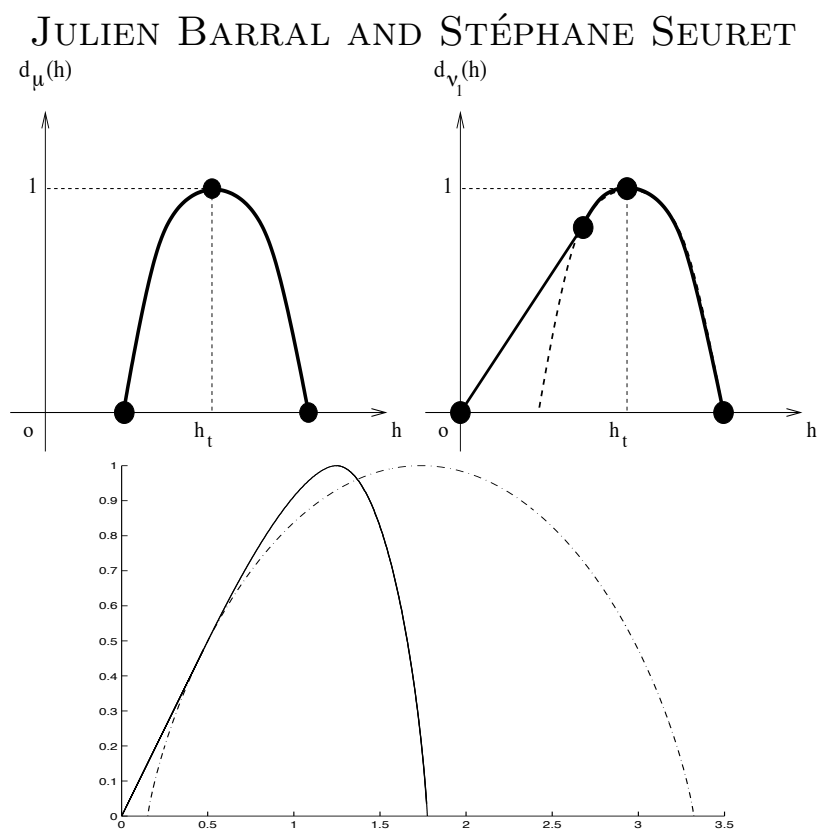

Fig. 1. Top left: Typical Hausdorff spectrum $d_{\mu}$ of a random self-similar measure $\mu$. Top right: Hausdorff spectrum $d_{\nu_{1}}$ of the corresponding sum of Dirac masses $\nu_{1}$ with $\rho=1$. Bottom: Hausdorff spectrum $d_{\mu}$ of the binomial measure $\mu$ with $p_{0}=1 / 10$ and $p_{1}=9 / 10$ (dashed graph), and Hausdorff spectrum $d_{\nu_{1 / 3}}$ of its associated sum of Dirac masses with $\rho=1 / 3$ (plain graph). The multifractal spectrum of $\nu_{1 / 3}$ is highly asymmetric.

(ii) If $h \geq h_{c}(\mu)$, then $d_{\nu_{1}}(h) \leq \tau_{\mu}^{*}(h)$ if $\tau_{\mu}^{*}(h) \geq 0$, and $E_{h}^{\nu_{1}}=\emptyset$ if $\tau_{\mu}^{*}(h)<0$. Moreover, if $\mathbf{P 3}(\mu, h)$ holds then $d_{\nu_{1}}(h)=\tau_{\mu}^{*}(h)$.

Case $\rho<1$.

(i) If $h_{c}(\mu)>0$, then $d_{\nu_{\rho}}(h) \leq h$ for every $h \in\left[0, h_{c}(\mu)\right]$.

Moreover, if $\mathbf{P 2}\left(\mu, \rho,\left\{\left(x_{n}, \lambda_{n}\right)\right\}, h_{c}(\mu)\right)$ holds, then $d_{\nu_{\rho}}(h)=h$ for every $h \in$ $\left[0, h_{c}(\mu)\right]$.

(ii) Consider the exponent

$$
h_{\rho}(\mu)=d(1-\rho)+\rho \tau_{\mu}^{\prime}\left(0^{+}\right)
$$

and the mapping

$$
\alpha \geq 0 \mapsto \zeta(\alpha)=\frac{d(1-\rho)+\rho \tau_{\mu}^{*}(\alpha)}{d(1-\rho)+\rho \alpha} .
$$

If $h \in\left(h_{c}(\mu), h_{\rho}(\mu)\right)$ (this interval is non-empty if and only if $\tau_{\mu}^{\prime}\left(0^{+}\right)>d$, or equivalently $\left.h_{c}(\mu)<d\right)$, there exists a unique $\alpha=\alpha(h) \in\left(h_{c}(\mu), \tau_{\mu}^{\prime}\left(0^{+}\right)\right)$such that $\tau_{\mu}^{*}(\alpha)=h \cdot \zeta(\alpha)$. Then $d_{\nu_{\rho}}(h) \leq \tau_{\mu}^{*}(\alpha(h))$.

Moreover, if P2 $\left(\mu, \rho,\left\{\left(x_{n}, \lambda_{n}\right)\right\}, \alpha(h)\right)$ holds then $d_{\nu_{\rho}}(h)=\tau_{\mu}^{*}(\alpha(h))$.

(iii) If $h \geq h_{\rho}(\mu)$, let $\beta(h)$ be the exponent

$$
\beta(h)=\frac{h-d(1-\rho)}{\rho} .
$$

Then $d_{\nu_{\rho}}(h) \leq \tau_{\mu}^{*}(\beta(h))$, and $E_{h}^{\nu_{\rho}}=\emptyset$ if $\tau_{\mu}^{*}(\beta(h))<0$.

Moreover, if $\mathbf{P 3}(\mu, \beta(h))$ holds, then $d_{\nu_{\rho}}(h)=\tau_{\mu}^{*}(\beta(h))$. 
The multifractal nature of heterogeneous sums of Dirac masses

Consequently, as claimed in the simpler context of Theorem 1.3, under some assumptions on $\mu$ and $\left\{\left(x_{n}, \lambda_{n}\right)\right\}$, when $h_{c}(\mu)>0$, the multifractal spectrum of $\nu_{\rho}$ is composed of two parts: a linear part (starting at $(0,0))$ when $h$ is smaller than the critical value $h_{c}(\mu)$, and then a concave part when $h \geq h_{c}(\mu)$. This is a mixture between the linear shape obtained in the homogeneous case (1.2) in Theorem 1.1 and the classical strictly concave spectrum of random self-similar measures obeying a multifractal formalism [13, 30].

The heterogeneous ubiquity is responsible for the linear part of the spectrum, and when $\rho<1$, it is also responsible for the value of the spectrum on the interval $\left(h_{c}(\mu), h_{\rho}(\mu)\right)$. We are able to prove that $h \mapsto \tau_{\mu}^{*}(\alpha(h))$ is concave on the interval $\left(h_{c}(\mu), h_{\rho}(\mu)\right)$ only when $d=1$.

Section 5 provides examples illustrating Theorem 2.9, including the case $h_{c}(\mu)=0$. The proof of Theorem 2.9 begins in Section 3, where the sets $E_{h}^{\nu_{\rho}}$ are characterized in terms of the measure $\mu$ in Theorem 3.2. The proof ends in Section 4 , where Theorem $3 \cdot 2$ is used to find an upper bound and a lower bound for $\operatorname{dim} E_{h}^{\nu_{\rho}}$.

It is natural to ask whether some multifractal formalism is satisfied by $\nu_{\rho}$ or not. This question is discussed in Section 6.

\section{Local regularity of $\nu_{\rho}$ and level sets of $h_{\nu_{\rho}}$}

Let $\mu$ be a positive Borel measure on $\Omega=[0,1]^{d}$ with $\operatorname{supp}(\mu)=\Omega$ and $c$ an integer $\geq 2$. A weakly redundant system $\left\{\left(x_{n}, \lambda_{n}\right)\right\}_{n}$ is also fixed. Using the weak redundancy assumption on $\left\{\left(x_{n}, \lambda_{n}\right)\right\}_{n}$, if $y \in S$, then (remember Definition $2 \cdot 1$ for the value of $n_{y}$ )

$$
\frac{\lambda_{n_{y}}^{d(1-\rho)} \mu\left(B\left(y, \lambda_{n_{y}}^{\rho}\right)\right)}{c_{n_{y}}\left|\log \lambda_{n_{y}}\right|^{2}} \leq \nu_{\rho}(\{y\}) \leq 4 \frac{\lambda_{n_{y}}^{d(1-\rho)} \mu\left(B\left(y, \lambda_{n_{y}}^{\rho}\right)\right)}{\left|\log \lambda_{n_{y}}\right|} .
$$

Definition 3.1. Let $\alpha \geq 0, \xi \geq 1$ be two real numbers. Let $\varepsilon>0$. For every point $x \in \Omega$, the property $\mathcal{P}(\rho, \alpha, \xi, \varepsilon)$ is said to hold at $x$ if there exist $\eta<\varepsilon$ and an infinite number of irreducible points $y \in S$ such that

$$
\lambda_{n_{y}}^{\rho(\alpha+\eta)} \leq \mu\left(B\left(y, \lambda_{n_{y}}^{\rho}\right)\right) \leq \lambda_{n_{y}}^{\rho(\alpha-\eta)} \text { and }\|x-y\|_{\infty} \leq \lambda_{n_{y}}^{\xi-\eta} .
$$

For $h \geq 0$, let

$$
\begin{aligned}
F_{h, \rho} & =\left\{x \in \Omega:\left\{\begin{array}{c}
\forall \varepsilon>0, \exists \alpha \geq 0, \xi \geq 1 \text { such that } \\
\frac{d(1-\rho)+\rho \alpha}{\xi} \leq h+\varepsilon \text { and } \mathcal{P}(\rho, \alpha, \xi, \varepsilon) \text { holds at } x
\end{array}\right\}\right. \\
G_{h, \rho} & =F_{h, \rho} \bigcup\left\{x \in \Omega: h_{\mu}(x) \leq \max (\beta(h), h)\right\} .
\end{aligned}
$$

It is immediate that for any $0 \leq h \leq h^{\prime}, F_{h, \rho} \subset F_{h^{\prime}, \rho}$ and $G_{h, \rho} \subset G_{h^{\prime}, \rho}$

The following result exhibits, for every $h>0$, two sets $A_{h}$ and $B_{h}$ such that $A_{h} \subset$ $E_{h}^{\nu_{\rho}} \subset B_{h}$. The sets $A_{h}$ and $B_{h}$ are used to find respectively a lower bound and an upper bound for $\operatorname{dim} E_{h}^{\nu_{\rho}}$.

Theorem 3.2. Assume that $\mathbf{P} \mathbf{1}(\mu)$ holds, and let $h>0$.

Then $A_{h} \subset E_{h}^{\nu_{\rho}} \subset B_{h}$, where

(i) If $h<h_{\rho}(\mu)$, then

$$
\left\{\begin{array}{l}
A_{h}=\left(\widetilde{E}_{\max (h, \beta(h))}^{\mu} \cap\left\{x \in \Omega: \xi_{x}=1\right\}\right) \cup\left(F_{h, \rho} \backslash \bigcup_{h^{\prime}<h} G_{h^{\prime}, \rho}\right), \\
B_{h}=G_{h, \rho} \backslash \bigcup_{h^{\prime}<h} F_{h^{\prime}, \rho} .
\end{array}\right.
$$


(ii) If $h \geq h_{\rho}(\mu)$, then

$$
\left\{\begin{array}{l}
A_{h}=\widetilde{E}_{\beta(h)}^{\mu} \cap\left\{x \in \Omega: \xi_{x}=1\right\} \\
B_{h}=\bigcup_{h^{\prime} \geq \beta(h)} \bar{E}_{h^{\prime}}^{\mu}
\end{array}\right.
$$

Theorem $3 \cdot 2$ is a consequence of Propositions $3 \cdot 3,3 \cdot 5$ and $3 \cdot 6$.

Proposition 3.3. Let $x \in \Omega \backslash S=\Omega \backslash\left\{x_{n}: n \geq 0\right\}$. If $\xi_{x}<+\infty$, then

$$
\min \left(\frac{d(1-\rho)+\rho h_{\mu}(x)}{\xi_{x}}, h_{\mu}(x)\right) \leq h_{\nu_{\rho}}(x) \leq \frac{d(1-\rho)+\rho \bar{h}_{\mu}(x)}{\xi_{x}} .
$$

Moreover, if $\mathbf{P 1}(\mu)$ holds and $\xi_{x}=+\infty$, then $h_{\nu_{\rho}}(x)=0$.

Proof. We denote $\alpha=h_{\mu}(x), \beta=\bar{h}_{\mu}(x)$. Let $\varepsilon>0$, and assume that $\xi_{x}<+\infty$.

We start with the upper bound for $h_{\nu_{\rho}}(x)$.

Assume first that $\xi_{x}>1$.

By definition of $\xi_{x}$ and by $\mathbf{P} \mathbf{4}$, there exists an infinite number of irreducible points $x_{p} \in S$ such that $\left\|x-x_{p}\right\|_{\infty} \leq \lambda_{n_{x_{p}}}^{\xi_{x}-\varepsilon} / 2$. Let $x_{p}$ be such a point. By $(3 \cdot 1)$,

$$
\nu_{\rho}\left(B\left(x, \lambda_{n_{x_{p}}}^{\xi_{x}-\varepsilon} / 2\right)\right) \geq \nu_{\rho}\left(\left\{x_{p}\right\}\right) \geq\left|\log \lambda_{n_{x_{p}}}\right|^{-2} c_{n_{x_{p}}}^{-1} \lambda_{n_{x_{p}}}^{d(1-\rho)} \mu\left(B\left(x_{p}, \lambda_{n_{x_{p}}}^{\rho}\right)\right) .
$$

By definition of $\beta=\bar{h}_{\mu}(x)$, there exists a scale $J_{1}$ such that

$$
0<r<2^{-J_{1}} \text { implies } \mu(B(x, r)) \geq r^{\beta+\varepsilon} \text {. }
$$

By construction, $B\left(x, \lambda_{n_{x_{p}}}^{\rho} / 2\right) \subset B\left(x_{p}, \lambda_{n_{x_{p}}}^{\rho}\right)$. Consequently, when $n_{x_{p}}$ is large enough, $\mu\left(B\left(x_{p}, \lambda_{n_{x_{p}}}^{\rho}\right)\right) \geq \mu\left(B\left(x, \lambda_{n_{x_{p}}}^{\rho} / 2\right)\right) \geq\left(\lambda_{n_{x_{p}}}^{\rho} / 2\right)^{\beta+\varepsilon}$. Hence

$$
\nu_{\rho}\left(B\left(x, \lambda_{n_{x_{p}}}^{\xi_{x}-\varepsilon} / 2\right)\right) \geq 2^{-(\beta+\varepsilon)} c_{n_{x_{p}}}^{-1}\left|\log \lambda_{n_{x_{p}}}\right|^{-2} \lambda_{n_{x_{p}}}^{d(1-\rho)+\rho(\beta+\varepsilon))}
$$

Because of the weak redundancy of $\left\{\left(x_{n}, \lambda_{n}\right)\right\}_{n}, \log c_{n_{x_{p}}}=o\left(\left|\log \lambda_{n_{x_{p}}}\right|\right)$. We thus deduce from (3.6) that $h_{\nu_{\rho}}(x) \leq \frac{d(1-\rho)+\rho(\beta+\varepsilon)}{\xi_{x}-\varepsilon}$. This remains true for every $\varepsilon>0$. Hence $h_{\nu_{\rho}}(x) \leq$ $\frac{d(1-\rho)+\rho \bar{h}_{\mu}(x)}{\xi_{x}}$.

If $\xi_{x}=1,\left\|x-x_{p}\right\|_{\infty} \leq \lambda_{n_{x_{p}}}^{\xi_{x}-\varepsilon} / 2$ above is replaced by $\left\|x-x_{p}\right\|_{\infty} \leq \lambda_{n_{x_{p}}} / 2$, and the previous lines show that $h_{\nu_{\rho}}(x) \leq d(1-\rho)+\rho \bar{h}_{\mu}(x)$. These lines also imply that if $\mathbf{P} \mathbf{1}(\mu)$ holds and $\xi_{x}=+\infty$, then $h_{\nu_{\rho}}(x)=0$.

Now we focus on the lower bound for $h_{\nu_{\rho}}(x)$. This part is more delicate to obtain. By definition of $\alpha=h_{\mu}(x)$, there exists $J_{2}$ such that

$$
0<r<2^{-J_{2}} \text { implies } \mu(B(x, r)) \leq r^{\alpha-\varepsilon} .
$$

By Proposition $2 \cdot 2$, there exists a scale $J_{3}$ such that for every $y \in S$ such that $\lambda_{n_{y}} \leq$ $2^{-J_{3}},\|x-y\|_{\infty} \geq \lambda_{n_{y}}^{\xi_{x}+\varepsilon}$. We can also take $J_{4} \geq J_{3}$ such that if $y \in S$ and $\lambda_{n_{y}} \leq 2^{-J_{4}}$ then $\forall y^{\prime} \in S$ such that $\lambda_{n_{y^{\prime}}} \geq 2^{-J_{3}}, y^{\prime} \notin B\left(x, \lambda_{n_{y}}\right)$.

In the rest of the proof, we often use the weak redundancy property (Definition 1.2) and the decomposition of the set $T_{j}$ into pairwise disjoint subsets $T_{j}=\bigcup_{i=1}^{N_{j}} T_{j, i}$, for some sequence $\left(N_{j}\right)_{j \geq 0}$ such that $\log N_{j}=o(j)$.

Let $j_{0} \geq 3 \xi_{x} \frac{\max \left(J_{2}, J_{4}\right)}{\rho}$. We now want to get an upper bound for $\nu_{\rho}\left(B\left(x, 2^{-j_{0}}\right)\right)$. This 
real number $\nu_{\rho}\left(B\left(x, 2^{-j_{0}}\right)\right)$ can be written as the sum $\sum_{j \geq 0} u_{j}(x)$, where

$$
u_{j}(x)=N_{j}^{-1} \sum_{n \in T_{j}} \mathbf{1}_{B\left(x, 2^{\left.-j_{0}\right)}\right.}\left(x_{n}\right) \frac{\mu\left(B\left(x_{n}, \lambda_{n}^{\rho}\right)\right)}{\lambda_{n}^{-d(1-\rho)}\left|\log \lambda_{n}\right|^{2}} .
$$

We split $\sum_{j \geq 0} u_{j}(x)$ into three terms studied below.

- Estimate of $\sum_{0 \leq j<j_{0}} u_{j}(x)$ : Suppose that this sum is not equal to 0 , and let $n_{0}=\min \left\{n: x_{n} \in \bigcup_{j<j_{0}}\left\{x_{p}: p \in T_{j}\right.\right.$ and $\left.\left.x_{p} \in B\left(x, 2^{-j_{0}}\right)\right\}\right\}$. Obviously, $n_{0}=n_{x_{n_{0}}}$, i.e. $x_{n_{0}}$ is its own irreducible form. This implies that $\lambda_{n_{0}}^{\xi_{x}+\varepsilon} \leq\left\|x-x_{n_{0}}\right\|_{\infty} \leq 2^{-j_{0}}<\lambda_{n_{0}}$. More generally, using that $j_{0} \geq 3 \xi_{x} \frac{\max \left(J_{2}, J_{4}\right)}{\rho}$, for every $y \in \bigcup_{j_{0} /\left(\xi_{x}+\varepsilon\right) \leq j<j_{0}}\left\{x_{n} \in B\left(x, 2^{-j_{0}}\right)\right.$ : $\left.n \in T_{j}\right\}$,

$$
\lambda_{n_{y}}^{\xi_{x}+\varepsilon} \leq\|x-y\|_{\infty} \leq 2^{-j_{0}}<\lambda_{n_{y}} .
$$

The previous remark yields

$$
\sum_{0 \leq j<j_{0}} u_{j}(x)=\sum_{j_{0} /\left(\xi_{x}+\varepsilon\right) \leq j<j_{0}} u_{j}(x) .
$$

Let $j_{0} /\left(\xi_{x}+\varepsilon\right) \leq j<j_{0}$ and $1 \leq i \leq N_{j}$. Using the weak redundancy property $T_{j}=$ $\bigcup_{i=1}^{N_{j}} T_{j, i}$, there is at most one integer element $n$ of $T_{j, i}$ such that $x_{n} \in B\left(x, 2^{-j}\right)$. Hence there are at most $N_{j}$ non-zero terms in $u_{j}(x)$.

For every $j_{0} /\left(\xi_{x}+\varepsilon\right) \leq j<j_{0}$ and $n \in T_{j}$ such that $x_{n} \in B\left(x, 2^{-j}\right)$, we have $B\left(x_{n}, \lambda_{n}^{\rho}\right) \subset B\left(x, 2 \cdot 2^{-j_{0} \rho /\left(\xi_{x}+\varepsilon\right)}\right)$. Thus each non-zero term in $u_{j}(x)$ is bounded by $N_{j}^{-1} 2^{-d(1-\rho) j} \mu\left(B\left(x, 2 \cdot 2^{-j_{0} \rho /\left(\xi_{x}+\varepsilon\right)}\right)\right)$. Consequently, using (3.7),

$$
\begin{aligned}
\sum_{j_{0} /\left(\xi_{x}+\varepsilon\right) \leq j<j_{0}} u_{j}(x) & \leq \mu\left(B\left(x, 2 \cdot 2^{-j_{0} \rho /\left(\xi_{x}+\varepsilon\right)}\right) \sum_{j_{0} /\left(\xi_{x}+\varepsilon\right) \leq j<j_{0}} 2^{-d(1-\rho) j}\right. \\
& \leq \mu\left(B\left(x, 2 \cdot 2^{-j_{0} \rho /\left(\xi_{x}+\varepsilon\right)}\right) j_{0} 2^{-d(1-\rho) j_{0} /\left(\xi_{x}+\varepsilon\right)}\right. \\
& \leq 2^{\alpha-\varepsilon} j_{0} 2^{-j_{0} \frac{d(1-\rho)+\rho(\alpha-\varepsilon))}{\xi_{x}+\varepsilon}} .
\end{aligned}
$$

- Estimate of $\sum_{j_{0} \leq j<j_{0} / \rho} u_{j}(x)$ : Let $j$ be an integer belonging to $\left[j_{0}, j_{0} / \rho\right]$. Fix $1 \leq i \leq N_{j}$. Applying again the weak redundancy property and the fact that the (disjoint) balls $B\left(x_{n}, \lambda_{n}\right), n \in T_{j, i}$, have radii ranging between $2^{-(j+1)}$ and $2^{-j}$, there exists a universal constant $C$ such that the number of elements $n$ of $T_{j, i}$ such that $x_{n} \in B\left(x, 2^{-j_{0}}\right)$ is bounded by $C 2^{d\left(j-j_{0}\right)}$. Moreover, for each such $n, \mu\left(B\left(x_{n}, \lambda_{n}^{\rho}\right)\right) \leq \mu\left(B\left(x, 2 \cdot 2^{-j \rho}\right)\right)$. Using $(3 \cdot 7)$ again, this yields

$$
\begin{aligned}
\sum_{j_{0} \leq j<j_{0} / \rho} u_{j}(x) & \leq \sum_{j_{0} \leq j<j_{0} / \rho} C 2^{d\left(j-j_{0}\right)} 2^{-d(1-\rho) j} 2^{\alpha-\varepsilon} 2^{-j \rho(\alpha-\varepsilon)} \\
& \leq C^{\prime} 2^{-d j_{0}} \sum_{j_{0} \leq j<j_{0} / \rho} 2^{j \rho(d-(\alpha-\varepsilon))} .
\end{aligned}
$$

If $\alpha>d$ and $\varepsilon$ is small enough, the last sum is bounded by $C 2^{-d j_{0}} 2^{j_{0} \rho(d-(\alpha-\varepsilon))} \leq$ $C 2^{-j_{0}(d(1-\rho)+\rho(\alpha-\varepsilon))}$.

If $\alpha \leq d$, the sum is bounded by $C 2^{-j_{0}(\alpha-\varepsilon)}$.

- Estimate of $\sum_{j>j_{0} / \rho} u_{j}(x)$ : The following result is needed.

LEMma 3.4. There exists a universal constant $C$ having the following property.

For every $y \in[0,1]^{d}$, for every $j_{0} \geq 0$ and for every $j>j_{0} / \rho$, let $\left\{B\left(y_{p}, r_{p}\right)\right\}_{p}$ be 
any family of pairwise disjoint balls of radii $r_{p}$ ranging from $2^{-(j+1)}$ to $2^{-j}$ such that $y_{p} \in B\left(y, 2^{-j_{0}}\right) \cap[0,1]^{d}$.

There exists an integer $N \leq C 2^{d(1-\rho) j}$ such that the family $\left\{B\left(y_{p}, r_{p}^{\rho}\right)\right\}_{p}$ can be partitioned into $N$ subfamilies of pairwise disjoint balls.

Proof. We use the dyadic cubes $I_{j, \mathbf{k}}^{2}$ of Section $2 \cdot 1$. Let $j \geq j_{0} / \rho$ and $j_{\rho}=[j \rho]$.

If $\mathbf{k} \in\{0,1\}^{d}$, let $A_{\mathbf{k}}=\left\{p: \exists \mathbf{k}^{\prime} \in\left\{0, \ldots 2^{j_{\rho}-1}-1\right\}^{d}, y_{p} \in I_{j_{\rho}, 2 \mathbf{k}^{\prime}+\mathbf{k}}^{2}\right\}$. Then let $\mathcal{B}_{\mathbf{k}}=$ $\left\{B\left(y_{p}, r_{p}^{\rho}\right): p \in A_{\mathbf{k}}\right\}$. By construction, the $2^{d}$ sets $\mathcal{B}_{\mathbf{k}}$ are pairwise disjoint and their union is equal to $\left\{B\left(y_{p}, r_{p}^{\rho}\right)\right\}_{p}$. Now, it remains to show that for each $\mathbf{k} \in\{0,1\}^{d}$, if $\mathcal{B}_{\mathbf{k}} \neq \emptyset$, this set can be partitioned into at most $C 2^{d(1-\rho) j}$ subfamilies of pairwise disjoint balls, for some universal constant $C$.

On the one hand, in every cube of the form $I_{j_{\rho}, 2 \mathbf{k}^{\prime}+\mathbf{k}}^{2}, \mathbf{k}^{\prime} \in\left\{0, \ldots 2^{j_{\rho}-1}-1\right\}^{d}$, there are at most $C 2^{d(1-\rho) j}$ balls $B\left(y_{p}, r_{p}\right)$, where $C$ is a constant depending only on $d$. Indeed, the volume of any of the disjoint balls $B\left(y_{p}, r_{p}\right), p \in \mathcal{A}_{\mathbf{k}}$, is greater than $2^{-d(j+1)}$, and the volume of the cube $I_{j_{\rho}, 2 \mathbf{k}^{\prime}+\mathbf{k}}^{2}$ is $2^{-d j_{\rho}}$.

On the other hand, if two points $y_{p_{1}} \in I_{j_{\rho}, 2 \mathbf{k}_{1}^{\prime}+\mathbf{k}}^{2}$ and $y_{p_{2}} \in I_{j_{\rho}, 2 \mathbf{k}_{2}^{\prime}+\mathbf{k}}^{2}$ with $\mathbf{k}_{1}^{\prime}, \mathbf{k}_{2}^{\prime} \in$ $\left\{0, \ldots, 2^{j_{\rho}-1}-1\right\}^{d}$ and $\mathbf{k}_{1}^{\prime} \neq \mathbf{k}_{2}^{\prime}$, then $B\left(y_{p_{1}}, r_{p_{1}}^{\rho}\right) \cap B\left(y_{p_{2}}, r_{p_{2}}^{\rho}\right)=\emptyset$. This makes it possible to split $\mathcal{B}_{\mathbf{k}}$ suitably.

Let $j>j_{0} / \rho$. This time, if $1 \leq i \leq N_{j}$, for every $n \in T_{j, i}$, the ball $B\left(x_{n}, \lambda_{n}^{\rho}\right)$ has a diameter smaller than $2 \cdot 2^{-j_{0}}$. Thus if the corresponding point $x_{n}$ belongs to $B\left(x, 2^{-j_{0}}\right)$, then $B\left(x_{n}, \lambda_{n}^{\rho}\right)$ is contained in $\widetilde{B}=B\left(x, 3 \cdot 2^{-j_{0}}\right)$.

Lemma $3 \cdot 4$ can be applied with $y=x$ and to $\left\{B\left(y_{p}, r_{p}\right)\right\}=\left\{B\left(x_{n}, \lambda_{n}^{\rho}\right): n \in\right.$ $T_{j, i}$ and $\left.x_{n} \in B\left(x, 2^{-j_{0}}\right)\right\}$. This family can be split into $N_{j, i, \rho} \leq P 2^{j d(1-\rho)}$ families of disjoint balls $\mathcal{B}_{j, i, k}$, and $\bigcup_{B \in \mathcal{B}_{j, i, k}} B \subset \widetilde{B}$. Hence

$$
\begin{aligned}
u_{j}(x) & =N_{j}^{-1} \sum_{n \in T_{j}} \mathbf{1}_{B\left(x, 2^{\left.-j_{0}\right)}\right.}\left(x_{n}\right) \frac{\mu\left(B\left(x_{n}, \lambda_{n}^{\rho}\right)\right)}{\lambda_{n}^{-d(1-\rho)}\left|\log \lambda_{n}\right|^{2}} \\
& \leq \frac{2^{-j d(1-\rho)}}{j^{2} \log ^{2}(2)} N_{j}^{-1} \sum_{i=1}^{N_{j}} \sum_{k=1}^{N_{j, i, \rho}} \sum_{B \in \mathcal{B}_{i, j, k}} \mu(B) \leq C j^{-2} \mu(\widetilde{B}),
\end{aligned}
$$

for another constant $C$. Consequently

$$
\sum_{j>j_{0} / \rho} u_{j}(x) \leq C j_{0}^{-1} \mu(\widetilde{B}) \leq P 2^{-j_{0}(\alpha-\varepsilon)} .
$$

As a consequence, if $\varepsilon$ is small enough, for $j_{0}$ large enough, we get:

- If $\alpha \leq d, \nu_{\rho}\left(B\left(x, 2^{-j_{0}}\right)\right) \leq C\left(2^{-j_{0} \frac{d(1-\rho)+\rho(\alpha-\varepsilon)}{\xi x+\varepsilon}}+2^{-j_{0}(\alpha-\varepsilon)}+2^{-j_{0}(\alpha-\varepsilon)}\right)$.

- If $\alpha>d, \nu_{\rho}\left(B\left(x, 2^{-j_{0}}\right)\right) \leq C\left(2^{-j_{0} \frac{d(1-\rho)+\rho(\alpha-\varepsilon)}{\xi_{x}+\varepsilon}}+2^{-j_{0}(d(1-\rho)+\rho(\alpha-\varepsilon))}+2^{-j_{0}(\alpha-\varepsilon)}\right)$.

In all these cases (and remarking that $d(1-\rho)+\rho \alpha$ is always greater than $\left.\frac{d(1-\rho)+\rho \alpha}{\xi_{x}}\right)$, letting $\varepsilon$ tend to zero gives Proposition $3 \cdot 3$.

Proposition 3.5. If $x \in F_{h, \rho}$ for some $h \geq 0$, then $h_{\nu_{\rho}}(x) \leq h$.

Proof. Let $\varepsilon>0, \alpha \geq 0$ and $\xi \geq 1$ such that $\frac{d(1-\rho)+\rho \alpha}{\xi} \leq h+\varepsilon$ and $\mathcal{P}(\rho, \alpha, \xi, \varepsilon)$ holds at $x$. Let $y \in S$ be such that (3.2) holds. Then $\nu_{\rho}\left(B\left(x, 2 \lambda_{n_{y}}^{\xi-\eta}\right)\right) \geq \nu_{\rho}(\{y\})$, and the proof ends as in the proof of the upper bound for $h_{\nu_{\rho}}(x)$ in Proposition $3 \cdot 3$.

Proposition 3.6. If $\mathbf{P} 1(\mu)$ holds, then $E_{h}^{\nu_{\rho}} \subset G_{h, \rho}$ for every $0<h \leq h_{\rho}(\mu)$. 
Proof. Let $x \in E_{h}^{\nu_{\rho}}$. Denote $\alpha_{x}=h_{\mu}(x), h_{\rho}=\max (\beta(h), h)$, and let $\varepsilon>0$. Assume that $\alpha_{x}>h_{\rho}$. We want to show that $x \in F_{h, \rho}$.

By definition of $E_{h}^{\nu_{\rho}}$, there exists a sequence of integers $\left(j_{p}\right)_{p \geq 0}$ such that

$$
\nu_{\rho}\left(B\left(x, 2^{-j_{p}}\right)\right) \geq 2^{-j_{p}(h+\varepsilon / 2)} .
$$

We fix the integers $J_{2}$ and $J_{4}$ as in the proof of Proposition 3.3. Using results obtained in the proof of Proposition $3 \cdot 3$, if $j_{p}$ is large enough, then

$$
\nu_{\rho}\left(B\left(x, 2^{-j_{p}}\right)\right) \leq C\left(2^{-j_{p}\left(d(1-\rho)+\rho\left(\alpha_{x}-\varepsilon\right)\right)}+2^{-j_{p}\left(\alpha_{x}-\varepsilon\right)}\right)+\sum_{j_{p} /\left(\xi_{x}+\varepsilon\right) \leq j<j_{p}} u_{j}(x) .
$$

Since $\alpha_{x}>h_{\rho}, \varepsilon$ can be chosen small enough so that $\min \left(d(1-\rho)+\rho\left(\alpha_{x}-\varepsilon\right), \alpha_{x}-\varepsilon\right)>$ $h+2 \varepsilon$. Using $(3 \cdot 10)$ and $(3 \cdot 11)$, we get for $j_{p}$ large enough

$$
2^{-j_{p}(h+\varepsilon)} \leq \sum_{j_{p} /\left(\xi_{x}+\varepsilon\right) \leq j<j_{p}} N_{j}^{-1} \sum_{n \in T_{j}} \mathbf{1}_{B\left(x, 2^{-j_{p}}\right)}\left(x_{n}\right) \frac{\mu\left(B\left(x_{n}, \lambda_{n}^{\rho}\right)\right)}{\lambda_{n}^{-d(1-\rho)}\left|\log \lambda_{n}\right|^{2}} .
$$

Let $n_{p}$ be minimal in the subset of $\bigcup_{j_{p} /\left(\xi_{x}+\varepsilon\right) \leq j<j_{p}} T_{j}$ consisting of the integers $k$ such that

$$
\frac{\mu\left(B\left(x_{k}, \lambda_{k}^{\rho}\right)\right)}{\lambda_{k}^{-d(1-\rho)}\left|\log \lambda_{k}\right|^{2}}=\max _{n \in \bigcup_{j_{p} /\left(\xi_{x}+\varepsilon\right) \leq j<j_{p}} T_{j}} \mathbf{1}_{B\left(x, 2^{-j_{p}}\right)}\left(x_{n}\right) \frac{\mu\left(B\left(x_{n}, \lambda_{n}^{\rho}\right)\right)}{\lambda_{n}^{-d(1-\rho)}\left|\log \lambda_{n}\right|^{2}} .
$$

If $x_{n}=x_{n^{\prime}}$ with $n^{\prime}>n$, then $\frac{\mu\left(B\left(x_{n}, \lambda_{n}^{\rho}\right)\right)}{\lambda_{n}^{-d(1-\rho)}\left|\log \lambda_{n}\right|^{2}} \geq \frac{\mu\left(B\left(x_{n^{\prime}}, \lambda_{n^{\prime}}^{\rho}\right)\right)}{\lambda_{n^{\prime}}^{-d(1-\rho)}\left|\log \lambda_{n^{\prime}}\right|^{2}}$. This fact and our choice of the integer $J_{4}$ together imply that the point $x_{n_{p}}$ is irreducible. Combining Proposition 3.3 and the definition of $n_{p}$, we get

$$
2^{-j_{p}(h+\varepsilon)} \leq \frac{\mu\left(B\left(x_{n_{p}}, \lambda_{n_{p}}^{\rho}\right)\right)}{\lambda_{n_{p}}^{-d(1-\rho)}\left|\log \lambda_{n_{p}}\right|^{2}} .
$$

Write $\left|x-x_{n_{p}}\right|=\lambda_{n_{p}}^{\xi_{p}} \leq \lambda_{n_{p}}$ with $\xi_{p} \geq 1$, and $\mu\left(B\left(x_{n_{p}}, \lambda_{n_{p}}^{\rho}\right)\right)=\lambda_{n_{p}}^{\rho \alpha_{p}}$ for some $\alpha_{p} \geq 0$. Using (3.12) and (3.8), this exponent $\alpha_{p}$ satisfies

$$
2^{-j_{p}(h+\varepsilon)} \leq \lambda_{n_{p}}^{d(1-\rho)+\rho \alpha_{p}} \leq 2^{-j_{p} \frac{d(1-\rho)+\rho \alpha_{p}}{\xi_{p}}},
$$

which implies $\frac{1-\rho+\rho \alpha_{p}}{\xi_{p}} \leq h+\varepsilon$.

Performing this analysis for every $p$ large enough gives us an infinite sequence of irreducible points $x_{n_{p}}$ that satisfy $\left|x-x_{n_{p}}\right|=\lambda_{n_{p}}^{\xi_{p}}$ and $\mu\left(B\left(x_{n_{p}}, \lambda_{n_{p}}^{\rho}\right)\right)=\lambda_{n_{p}}^{\rho \alpha_{p}}$ with $\left(\xi_{p}, \alpha_{p}\right) \in\left[1, \xi_{x}+1\right] \times[0, M]$. Indeed, by construction $\xi_{p} \leq \xi_{x}+1$ for $p$ large enough, and $M$ is the upper bound implied by the verification of condition $\mathbf{P} 1(\mu)$. Thus, up to a subsequence, $\left(\xi_{p}, \alpha_{p}\right)$ converges to $(\xi, \alpha) \in\left[1, \xi_{x}+1\right] \times[0, M]$.

Since $\frac{1-\rho+\rho \alpha_{p}}{\xi_{p}} \leq h+\varepsilon$ for every $p$, the pair $(\xi, \alpha)$ also satisfies this inequality, and by construction $x$ satisfies $\mathcal{P}(\rho, \alpha, \xi, \varepsilon)$. Finally $x \in F_{h, \rho}$.

\section{Computation of the spectrum of $\nu_{\rho}$ : Proof of Theorem 2.9}

As above, the system $\left\{\left(x_{n}, \lambda_{n}\right)\right\}_{n}$ is always supposed to be weakly redundant. 


\subsection{Preliminary results}

\section{$4 \cdot 1 \cdot 1$. Some Large Deviation estimates}

Definition $4 \cdot 1$. The function $\tau_{\mu, \rho}$ is defined by

$$
\tau_{\mu, \rho}(q)=\liminf _{j \rightarrow+\infty}-\frac{1}{j} \log _{2} \sum_{n \in T_{j}} \mu\left(B\left(x_{n}, \lambda_{n}^{\rho}\right)\right)^{q} .
$$

It is established in $[\mathbf{1 1}]$ that if the system $\left\{\left(x_{n}, \lambda_{n}\right)\right\}_{n}$ is weakly redundant, then

$$
\forall q \in \mathbb{R}, \tau_{\mu, \rho}(q) \geq d(1-\rho)+\rho \tau_{\mu}(q) .
$$

The following result is a consequence of some Tchernoff inequalities and $(4 \cdot 2)$.

LemMA $4 \cdot 2$. For every $0 \leq \beta \leq \alpha$ and for every $\varepsilon>0$, there exists a scale $J$ such that $j \geq J$ implies

$$
\frac{\log _{2} \#\left\{n \in T_{j}: \lambda_{n}^{-\rho \alpha} \leq \mu\left(B\left(x_{n}, \lambda_{n}^{\rho}\right)\right) \leq \lambda_{n}^{-\rho \beta}\right\}}{j} \leq d(1-\rho)+\rho \sup _{\beta \leq \alpha^{\prime} \leq \alpha} \tau_{\mu}^{*}\left(\alpha^{\prime}\right)+\varepsilon .
$$

Tchernoff inequalities also yield the following lemma, in which $c$ is any integer $\geq 2$.

Lemma $4 \cdot 3$. Let $0 \leq \beta \leq \alpha, M>1$ and $\varepsilon>0$. There exists a scale $J$ such that

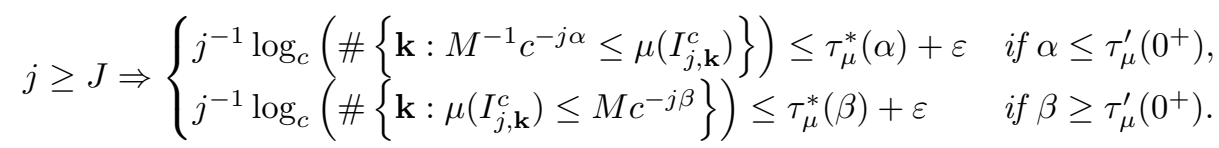

\section{$4 \cdot 1 \cdot 2$. Upper bound for the Hausdorff dimension of union of level sets}

The following proposition is a consequence of $[\mathbf{1 3}],[\mathbf{3 0}],[\mathbf{2 5}]$ and $[\mathbf{5}]$. The results concerning the set $\widetilde{E}_{\alpha}^{\mu}$ defined in Section $2 \cdot 1$ are valid in any basis $c \geq 2$.

Proposition 4.4. Let $\alpha \geq 0$.

(i) If $\tau_{\mu}^{*}(\alpha)<0$, then $\widetilde{E}_{\alpha}^{\mu}=E_{\alpha}^{\mu}=\emptyset$.

(ii) $\tilde{d}_{\mu}(\alpha) \leq \tau_{\mu}^{*}(\alpha)$.

(iii) If $\alpha \in\left[0, \tau_{\mu}^{\prime}\left(0^{+}\right)\right]$, then $\operatorname{dim} \bigcup_{\alpha^{\prime} \leq \alpha} E_{\alpha^{\prime}}^{\mu} \leq \tau_{\mu}^{*}(\alpha)$.

(iv) If $\alpha \geq \tau_{\mu}^{\prime}\left(0^{+}\right)$, then $\operatorname{dim} \bigcup_{\alpha^{\prime} \geq \alpha} \bar{E}_{\alpha^{\prime}}^{\mu} \leq \tau_{\mu}^{*}(\alpha)$.

\section{$4 \cdot 1 \cdot 3$. Upper bound for the Hausdorff dimension of some limsup sets}

We introduce, for $0 \leq \beta \leq \alpha$ and $\xi \geq 1$, the sets

$$
S_{\mu}(\rho, \alpha, \beta, \xi)=\bigcap_{N \geq 0} \bigcup_{n \geq N: \lambda_{n}^{\rho \alpha} \leq \mu\left(B\left(x_{n}, \lambda_{n}^{\rho}\right)\right) \leq \lambda_{n}^{\rho \beta}} B\left(x_{n}, \lambda_{n}^{\xi}\right) .
$$

These sets are useful to find an upper bound for the spectrum of $\nu_{\rho}$.

LEMMA 4.5. Let $\left\{\left(x_{n}, \lambda_{n}\right)\right\}_{n}$ be a weakly redundant system. For every $\rho \in(0,1], 0 \leq$ $\beta \leq \alpha$ and $\xi \geq 1$

$$
\operatorname{dim} S_{\mu}(\rho, \alpha, \beta, \xi) \leq \min \left(\sup _{\beta \leq \alpha^{\prime} \leq \alpha} \tau_{\mu}^{*}\left(\alpha^{\prime}\right), \frac{d(1-\rho)+\rho \sup _{\beta \leq \alpha^{\prime} \leq \alpha} \tau_{\mu}^{*}\left(\alpha^{\prime}\right)}{\xi}\right)
$$

Proof. We first show that $\operatorname{dim} S_{\rho, \alpha, \beta, \xi} \leq \sup _{\beta \leq \alpha^{\prime} \leq \alpha} \tau_{\mu}^{*}\left(\alpha^{\prime}\right)$.

If $\beta \leq \tau_{\mu}^{\prime}\left(0^{+}\right) \leq \alpha$, there is nothing to prove since $\sup _{\beta \leq \alpha^{\prime} \leq \alpha} \tau_{\mu}^{*}\left(\alpha^{\prime}\right)=d$.

Let $j \geq 0$ and $n \in T_{j}$ such that $\mu\left(B\left(x_{n}, \lambda_{n}^{\rho}\right)\right) \in\left[\lambda_{n}^{\rho \alpha}, \lambda_{n}^{\rho \beta}\right]$. Let $j_{\rho}=[(j+1) \rho]+1$. By 
construction, there exists a dyadic cube $I_{\rho}$ of generation $j_{\rho}$ such that $I_{\rho} \cap B\left(x_{n}, \lambda_{n}^{\rho}\right) \neq \emptyset$ and $\mu\left(I_{\rho}\right) \geq 2^{-j_{\rho} \alpha} / 4^{d}$. The cube $I_{\rho}$ can be written $I_{j_{\rho}, \mathbf{k}_{\rho}}^{2}$ (defined in Section $2 \cdot 1$ ) for some multi-integer $\mathbf{k}_{\rho} \in\left\{0, \ldots, 2^{j_{\rho}}-1\right\}^{d}$. Also, there exists $\mathbf{k}_{\rho}^{\prime} \in\{-8, . ., 0, . ., 8\}^{d}$ such that $I_{j_{\rho}, \mathbf{k}_{\rho}+\mathbf{k}_{\rho}^{\prime}}^{2} \subset B\left(x_{n}, \lambda_{n}^{\rho}\right)$, and thus $\mu\left(I_{j_{\rho}, \mathbf{k}_{\rho}+\mathbf{k}_{\rho}^{\prime}}^{2}\right) \leq 2^{-j_{\rho} \beta}$. Moreover, $B\left(x_{n}, \lambda_{n}^{\rho}\right) \subset$ $\bigcup_{\mathbf{k} \in\{-8, \ldots, 0, \ldots, 8\}^{d}} I_{j_{\rho}, \mathbf{k}_{\rho}+\mathbf{k}}^{2}$. It follows that

$$
\begin{aligned}
& S_{\mu}(\rho, \alpha, \beta, \xi) \subset \limsup _{j \rightarrow \infty} \bigcup_{\mathbf{k} \in\left\{0, \ldots, 2^{j}-1\right\}^{d}: \frac{2^{-j \alpha}}{4} \leq \mu\left(I_{j, \mathbf{k}}^{2}\right)} \bigcup_{\mathbf{k}^{\prime} \in\{-8, \ldots, 0, \ldots, 8\}^{d}} I_{j, \mathbf{k}+\mathbf{k}^{\prime}}^{2} \\
& S_{\mu}(\rho, \alpha, \beta, \xi) \subset \limsup _{j \rightarrow \infty} \bigcup_{\mathbf{k} \in\left\{0, \ldots, 2^{j}-1\right\}^{d}: \mu\left(I_{j, \mathbf{k}}^{2}\right) \leq 2^{-j \beta}} \bigcup_{\mathbf{k}^{\prime} \in\{-16, \ldots, 0, \ldots, 16\}^{d}}^{2} I_{j, \mathbf{k}+\mathbf{k}^{\prime}} \cdot
\end{aligned}
$$

Suppose that $\alpha \leq \tau_{\mu}^{\prime}\left(0^{+}\right)$. Then $\sup _{\beta \leq \alpha^{\prime} \leq \alpha} \tau_{\mu}^{*}\left(\alpha^{\prime}\right)=\sup _{\alpha^{\prime} \leq \alpha} \tau_{\mu}^{*}\left(\alpha^{\prime}\right)=\tau_{\mu}^{*}(\alpha)$. Let $\varepsilon>0$. By Lemma $4 \cdot 3$, for $j$ large enough,

$$
\frac{\log \#\left\{\mathbf{k}: \frac{2^{-j \alpha}}{4} \leq \mu\left(I_{j, \mathbf{k}}^{2}\right)\right\}}{\log 2^{j}} \leq d_{\varepsilon}
$$

where $d_{\varepsilon}=\tau_{\mu}^{*}(\alpha)+\varepsilon$. Let $\gamma>d_{\varepsilon}$. For every $j \geq 1$, let

$$
s_{j}:=\sum_{\left.k \in\left\{0, \ldots, 2^{j}-1\right\}^{d}: \frac{2^{-j \alpha}}{4} \leq \mu\left(I_{j, \mathbf{k}}^{2}\right)\right) \leq 4 \cdot 2^{-j \beta}} \sum_{\mathbf{k}^{\prime} \in\{-8, \ldots, 0, \ldots, 8\}^{d}}\left|I_{j, \mathbf{k}+\mathbf{k}^{\prime}}^{2}\right|^{\gamma} .
$$

We remark that $s_{j} \leq 17^{d} 2^{j d_{\varepsilon}} 2^{-j \gamma}$, thus $\sum_{j \geq 1} s_{j}<\infty$. Combining this with (4.4) yields the vanishing of the $\gamma$-dimensional Hausdorff measure of $S_{\mu}(\rho, \alpha, \beta, \xi)$. Hence for every $\varepsilon>0, \operatorname{dim} S_{\mu}(\rho, \alpha, \beta, \xi) \leq d_{\varepsilon}$. Letting $\varepsilon$ tend to zero yields $\operatorname{dim} S_{\mu}(\rho, \alpha, \beta, \xi) \leq \tau_{\mu}^{*}(\alpha)$.

The case $\tau_{\mu}^{\prime}\left(0^{+}\right)<\beta \leq \alpha$ is treated similarly by using (4.5).

The upper bound $\operatorname{dim} S_{\mu}(\rho, \alpha, \beta, \xi) \leq\left(d(1-\rho)+\rho \sup _{\beta \leq \alpha^{\prime} \leq \alpha} \tau_{\mu}^{*}\left(\alpha^{\prime}\right)\right) / \xi$ is a simple consequence of the definition of $S_{\mu}(\rho, \alpha, \beta, \xi)$ and Lemma $4 \cdot 2$.

4.2. Upper bound for the multifractal spectrum of $\nu_{\rho}$

We first take care of the decreasing part of $d_{\nu_{\rho}}$.

Proposition 4.6. Assume that $\mathbf{P 1}(\mu)$ holds. If $h \geq h_{\rho}(\mu)$, then $\operatorname{dim} E_{h}^{\nu_{\rho}} \leq \tau_{\mu}^{*}(\beta(h))$.

Proof. This is a consequence of Theorem 3.2(ii) and Proposition 4.4(iv).

The increasing part is more delicate.

Proposition 4.7. Assume that $\mathbf{P 1}(\mu)$ holds. Let $0<h<h_{\rho}(\mu)$. If $h<d$, then

$$
\operatorname{dim} E_{h}^{\nu_{\rho}} \leq \max \left(\tau_{\mu}^{*}(h), \sup _{\alpha \geq h} \min \left(\tau_{\mu}^{*}(\alpha), h \zeta(\alpha)\right)\right),
$$

(recall that $\zeta(\alpha)$ is defined by (2.10)) and if $h \geq d$, then

$$
\operatorname{dim} E_{h}^{\nu_{\rho}} \leq \max \left(\tau_{\mu}^{*}(\beta(h)), \sup _{\alpha \geq \beta(h)} \min \left(\tau_{\mu}^{*}(\alpha), h \zeta(\alpha)\right)\right) .
$$

Proof. By Theorem 3.2(i), $E_{h}^{\nu_{\rho}} \subset F_{h, \rho} \bigcup\left\{x \in \Omega: h_{\mu}(x) \leq \max (\beta(h), h)\right\}$.

The case $h \in\left[d, h_{\rho}(\mu)\right)$ : Here $\max (h, \beta(h))=\beta(h)$.

By Proposition $4 \cdot 4, \operatorname{dim}\left\{x \in \Omega: h_{\mu}(x) \leq \beta(h)\right\} \leq \tau_{\mu}^{*}(\beta(h))$. It remains to find an upper bound for the Hausdorff dimension of $F_{h, \rho}$.

Fix $0<\varepsilon \leq 1$. For every $i \in \mathbb{N}$, let $\xi_{i}=1+i \varepsilon$ and let $\alpha_{i}=\xi_{i} \frac{(h+\varepsilon)-d(1-\rho)}{\rho}$. 
Let $x \in F_{h, \rho}$. There exists a pair $(\alpha, \xi)$ and $\eta<\varepsilon$ such that (3.2) holds. Let $i_{x}$ be the unique integer so that $\xi \in\left[\xi_{i_{x}}, \xi_{i_{x}+1}\right)$. By construction,

$$
\alpha \leq \frac{\xi(h+\varepsilon)-d(1-\rho)}{\rho} \leq \alpha_{i_{x}}+\frac{\varepsilon(h+\varepsilon)}{\rho} .
$$

So $\alpha+\varepsilon \leq \alpha_{i_{x}}+M_{h} \varepsilon$, where $M_{h}=1+(h+1) / \rho$. Since (3.2) holds, this implies that $x \in S_{\mu}\left(\rho, \alpha_{i_{x}}+M_{h} \varepsilon, 0, \xi_{i}\right)$.

As a consequence, $F_{h, \rho} \subset \bigcup_{i \in \mathbb{N}} S_{\mu}\left(\rho, \widetilde{\alpha}_{i}, 0, \xi_{i}\right)$, where $\widetilde{\alpha}_{i}=\alpha_{i}+M_{h} \varepsilon$. This implies by Lemma 4.5 that

$$
\begin{aligned}
\operatorname{dim} F_{h, \rho} & \leq \sup _{i \in \mathbb{N}} \operatorname{dim} S_{\mu}\left(\rho, \widetilde{\alpha}_{i}, 0, \xi_{i}\right) \\
& \leq \sup _{i \in \mathbb{N}} \min \left(\sup _{\alpha^{\prime} \leq \widetilde{\alpha}_{i}} \tau_{\mu}^{*}\left(\alpha^{\prime}\right), \frac{d(1-\rho)+\rho \sup _{\alpha^{\prime} \leq \widetilde{\alpha}_{i}} \tau_{\mu}^{*}\left(\alpha^{\prime}\right)}{\xi_{i}}\right) .
\end{aligned}
$$

Since $(h+\varepsilon) \xi_{i}=d(1-\rho)+\rho \alpha_{i}$, we get

$$
\operatorname{dim} F_{h, \rho} \leq \sup _{i \in \mathbb{N}} \min \left(\sup _{\alpha^{\prime} \leq \widetilde{\alpha}_{i}} \tau_{\mu}^{*}\left(\alpha^{\prime}\right),(h+\varepsilon) \frac{d(1-\rho)+\rho \sup _{\alpha^{\prime} \leq \widetilde{\alpha}_{i}} \tau_{\mu}^{*}\left(\alpha^{\prime}\right)}{d(1-\rho)+\rho \alpha_{i}}\right) .
$$

This upper bound remains true when $\varepsilon \rightarrow 0$. Then, using the continuity of the Legendre transform $\tau_{\mu}^{*}$ on the interval where it is finite and remembering that $\alpha_{i} \geq \beta(h)$, we get

$$
\operatorname{dim} F_{h, \rho} \leq \sup _{\alpha \geq \beta(h)} \min \left(\sup _{\alpha^{\prime} \leq \alpha} \tau_{\mu}^{*}\left(\alpha^{\prime}\right), h \frac{d(1-\rho)+\rho \sup _{\alpha^{\prime} \leq \alpha} \tau_{\mu}^{*}\left(\alpha^{\prime}\right)}{d(1-\rho)+\rho \alpha}\right) .
$$

The result follows from the fact that $\tau_{\mu}^{*}$ is non-decreasing on $\left[0, \tau_{\mu}^{\prime}\left(0^{+}\right)\right]$and non-increasing on $\left[\tau_{\mu}^{\prime}\left(0^{+}\right), \infty\right)$.

The case $h<1$ : Similar computations apply here, except that $\alpha_{i}$ has to be taken larger than or equal to $h$ (instead of $\beta(h)$ ).

\subsection{Simplification of Proposition 4.7.}

We write $\alpha_{\min }=\inf \left\{\alpha: \tau_{\mu}^{*}(\alpha) \geq 0\right\}$. Remember that by $\mathbf{P 5}(\mu), q_{c}(\mu)=1$ and $\tau_{\mu}^{*}\left(h_{c}(\mu)\right)=h_{c}(\mu)$. Hence $\zeta\left(h_{c}(\mu)\right)=1$. We also point out the fact that $h_{c}(\mu)=\tau_{\mu}^{\prime}\left(0^{+}\right)$ if and only if $\tau_{\mu}^{\prime}\left(0^{+}\right)=d$ (i.e. $\tau_{\mu}$ is affine between 0 and 1 ), and the interval $\left(h_{c}(\mu), h_{\rho}(\mu)\right)$ is then empty.

\section{Proposition $4 \cdot 8$.}

(i) For every $h \in\left[0, h_{c}(\mu)\right]$, the upper bound given by Proposition 4.7 equals $h$.

(ii) Suppose that $\tau_{\mu}^{\prime}\left(0^{+}\right)>d$. For every $h \in\left(h_{c}(\mu), h_{\rho}(\mu)\right)$, there exists a unique $\alpha=\alpha(h) \in\left(h_{c}(\mu), h_{\rho}(\mu)\right]$ such that $\tau_{\mu}^{*}(\alpha)=h \zeta(\alpha)$, and the upper bound of Proposition 4.7 equals $\tau_{\mu}^{*}(\alpha(h))$.

(iii) Suppose that $\tau_{\mu}^{\prime}\left(0^{+}\right)>d$. Let

$$
\begin{gathered}
\theta: h \mapsto\left\{\begin{array}{ll}
h & \text { if } h \in\left[0, h_{c}(\mu)\right] \\
\tau_{\mu}^{*}(\alpha(h)) & \text { if } h \in\left(h_{c}(\mu), h_{\rho}(\mu)\right]
\end{array} \text { and } \bar{\theta}: h \mapsto \begin{cases}\tau_{\mu}^{*}(h) & \text { if } h \leq d \\
\tau_{\mu}^{*}(\beta(h)) & \text { if } h \in\left(d, h_{\rho}(\mu)\right] .\end{cases} \right. \\
\text { If } \rho=1 \text { then } \theta=\bar{\theta} \text { on }\left(h_{c}(\mu), h_{\rho}(\mu)\right) . \\
\text { If } \rho<1 \text { then } \theta>\bar{\theta} \text { on }\left(h_{c}(\mu), h_{\rho}(\mu)\right), \text { and } \theta \text { is concave increasing if } d=1 .
\end{gathered}
$$

Proof. Since $\tau_{\mu}^{*}(\alpha) \leq \alpha$ for every $\alpha \geq 0$, we have $\zeta(\alpha) \leq \zeta\left(h_{c}(\mu)\right)=1$. 
(i) Let $h \in\left[0, h_{c}(\mu)\right]$. The mapping $\alpha \mapsto \tau_{\mu}^{*}(\alpha)$ is increasing on $\left[\alpha_{\min }, h_{c}(\mu)\right]$, and $\tau_{\mu}^{*}\left(h_{c}(\mu)\right)=h_{c}(\mu) \geq h=h \zeta\left(h_{c}(\mu)\right)$. Hence the upper bound of Proposition 4.7 is $h$.

(ii) Let $h \in\left(h_{c}(\mu), h_{\rho}(\mu)\right)$. On one side, the function $f: \alpha \mapsto h \zeta(\alpha)$ is non-decreasing on $\left[0, h_{c}(\mu)\right]$ and decreasing on $\left(h_{c}(\mu), \tau_{\mu}^{\prime}\left(0^{+}\right)\right]$, and $f\left(h_{c}(\mu)\right)=h>h_{c}(\mu)$. On the other side, the mapping $\alpha \mapsto \tau_{\mu}^{*}(\alpha)$ is increasing on $\left[0, \tau_{\mu}^{\prime}\left(0^{+}\right)\right]$and $\tau_{\mu}^{*}\left(h_{c}(\mu)\right)=h_{c}(\mu)$. Since the functions $f$ and $\tau_{\mu}^{*}$ are continuous, there exists a unique exponent $\alpha(h) \in\left(h_{c}(\mu), \tau_{\mu}^{\prime}\left(0^{+}\right)\right)$ such that $\tau_{\mu}^{*}(\alpha(h))=h \zeta(\alpha(h))$.

If $\rho=1$, then $\forall h \in\left(h_{c}(\mu), h_{\rho}(\mu)\right), \alpha(h)=h$ (and $\left.\theta(h)=\bar{\theta}(h)\right)$.

Let $\rho \in(0,1)$. To see that $\bar{\theta}(h)<\theta(h)$ on $\left(h_{c}(\mu), d\right]$, we show that $h<\alpha(h)$ on this interval. This is equivalent to $\zeta(h)>\tau_{\mu}^{*}(h) / h$. This holds true because when $h \geq h_{c}(\mu)$ the mapping $h \mapsto \tau_{\mu}^{*}(h) / h$ is decreasing and the function $\zeta$ is increasing, and both functions coincide at $h_{c}(\mu)$.

To see that $\bar{\theta}(h)<\theta(h)$ on $\left(d, h_{\rho}(\mu)\right)$, we prove that $\beta(h)<\alpha(h)$ on this interval. This is equivalent to $d(1-\rho)+\rho \tau_{\mu}^{*}(\beta(h))>\tau_{\mu}^{*}(\beta(h))$. This last inequality is true, since $\beta(h)<\tau_{\mu}^{\prime}\left(0^{+}\right)$, which implies $\tau_{\mu}^{*}(\beta(h))<d$.

Consequently, by definition of $\alpha(h)$, the upper bound of Proposition 4.7 equals $\theta(h)=$ $\tau_{\mu}^{*}(\alpha(h))$ for all $h \in\left(h_{c}(\mu), h_{\rho}(\mu)\right)$.

(iii) It remains to show that the function $\theta$ is concave if $d=1$. In fact it is easier to show that its inverse function $\theta^{-1}$ is convex.

Assume that $\tau_{\mu}^{*}$ is twice differentiable on $\left(h_{c}(\mu), h_{\rho}(\mu)\right)$. The mapping $\left(\tau_{\mu}^{*}\right)^{-1}$ is well defined since $\tau_{\mu}^{*}$ is continuous and strictly increasing when $h \leq \tau_{\mu}^{\prime}\left(0^{+}\right)$. By definition, if $u=\theta(h)$ for some $h \in\left[h_{c}(\mu), h_{\rho}(\mu)\right]$, then

$$
\theta^{-1}(u)=u \frac{d(1-\rho)+\rho\left(\tau_{\mu}^{*}\right)^{-1}(u)}{d(1-\rho)+\rho u} .
$$

A computation shows that on $\left[h_{c}(\mu), h_{\rho}(\mu)\right)$, the second derivative of $\theta^{-1}$ is larger than a positive multiple of

$$
d(1-\rho)\left[\left(\left(\tau_{\mu}^{*}\right)^{-1}\right)^{\prime}(u)-\left(\tau_{\mu}^{*}\right)^{-1}(u)\right]+\rho\left[u\left(\left(\tau_{\mu}^{*}\right)^{-1}\right)^{\prime}(u)-\left(\tau_{\mu}^{*}\right)^{-1}(u)\right] .
$$

This term is non-negative if $d=1$. Indeed, on the interval $\theta\left(\left[h_{c}(\mu), h_{\rho}(\mu)\right)\right)$, the function $\left(\left(\tau_{\mu}^{*}\right)^{-1}\right)^{\prime}(u)$ is non-decreasing, $\left(\left(\tau_{\mu}^{*}\right)^{-1}\right)^{\prime}(u) \geq 1$, and $\left(\tau_{\mu}^{*}\right)^{-1}\left(h_{c}(\mu)\right)=h_{c}(\mu)$. So $\theta^{-1}$ is convex. The function $\theta$ remains concave near the intermediate point $h_{c}(\mu)$ since by construction $d_{\nu_{\rho}}(h) \leq h$.

The case where $\tau_{\mu}^{*}$ is non-differentiable is obtained by approaching uniformly from above $\tau_{\mu}^{*}$ on the interval $\left[h_{c}(\mu), h_{\rho}(\mu)\right]$ by increasing concave functions that are twice differentiable with derivatives smaller than or equal to 1.

Remark: The upper bound given by Proposition 4.7 also applies when $h \geq h_{\rho}(\mu)$, since in this case

$$
\sup _{\alpha \geq \beta(h)} \min \left(\tau_{\mu}^{*}(\alpha), h \zeta(\alpha)\right)=\tau_{\mu}^{*}(\beta(h)) .
$$

4.4. Lower bound for the spectrum of $\nu_{\rho}$

We consider the decreasing part.

Proposition 4.9. Let $h \geq h_{\rho}(\mu)$. If $\tau_{\mu}^{*}(\beta(h))>0$ and if $\mathbf{P 3}(\mu, \beta(h))$ is satisfied, then $d_{\nu_{\rho}}(h) \geq \tau_{\mu}^{*}(\beta(h))$. 
Proof. Let $m_{\beta(h)}$ be the measure given by $\mathbf{P 3}(\mu, \beta(h))$. Using Theorem $3 \cdot 2($ ii), it is enough to show that $\operatorname{dim} \widetilde{E}_{\beta(h)}^{\mu} \cap\left\{x \in \Omega: \xi_{x}=1\right\} \geq \tau_{\mu}^{*}(\beta(h))$, or equivalently by $\mathbf{P 3}(\mu, \beta(h))$ that $m_{\beta(h)}\left(\widetilde{E}_{\beta(h)}^{\mu} \cap\left\{x \in \Omega: \xi_{x}>1\right\}\right)=0$.

Let $\varepsilon>0, \eta>0$ and $x \in \widetilde{E}_{\beta(h)}^{\mu} \backslash\left\{x_{n}: n \geq 1\right\}$. If $\xi_{x}>1+\eta$, then there exist infinitely many $y \in\left\{x_{n}: n \geq 1\right\}$ such that $\|x-y\|_{\infty} \leq \lambda_{n_{y}}^{\xi_{x}-\eta / 2} \leq \lambda_{n_{y}}$. By definition of $\widetilde{E}_{\beta(h)}^{\mu}$, this implies that for such a point $y$, if $n_{y}$ is large enough, then $\lambda_{n_{y}}^{\beta(h)+\varepsilon} \leq \mu\left(B\left(y, \lambda_{n_{y}}\right)\right) \leq$ $\lambda_{n_{y}}^{\beta(h)-\varepsilon}$.

Thus, if $K_{\beta(h), 1+\eta}$ is the set $\left(\widetilde{E}_{\beta(h)}^{\mu} \backslash\left\{x_{n}: n \geq 1\right\}\right) \cap\left\{x: \xi_{x}>1+\eta\right\}$, then $K_{\beta(h), 1+\eta} \subset$ $S_{\mu}(1, \beta(h)+\varepsilon, \beta(h)-\varepsilon, 1+\eta / 2)$. Lemma 4.5 yields that $\operatorname{dim} S_{\mu}(1, \beta(h)+\varepsilon, \beta(h)-\varepsilon, 1+$ $\eta / 2) \leq \sup _{\beta(h)-\varepsilon \leq \alpha^{\prime} \leq \beta(h)+\varepsilon} \tau_{\mu}^{*}\left(\alpha^{\prime}\right) /(1+\eta / 2)$. Since this is true for any $\varepsilon>0$, the last inequality yields that $\operatorname{dim} K_{\beta(h), 1+\eta} \leq \tau_{\mu}^{*}(\beta(h)) /(1+\eta / 2)$ and that $m_{\beta(h)}\left(K_{\beta(h), 1+\eta}\right)=0$ (by $\mathbf{P 3}(\mu, \beta(h)))$.

Since $\left(\widetilde{E}_{\beta(h)}^{\mu} \backslash\left\{x_{n}: n \geq 1\right\}\right) \cap\left\{x: \xi_{x}>1\right\}$ is equal to the countable union $\bigcup_{i \geq 1} K_{\beta(h), 1+1 / i}$ and $\left\{x_{n}: n \geq 1\right\}$ is countable, $m_{\beta(h)}\left(\widetilde{E}_{\beta(h)}^{\mu} \cap\left\{x: \xi_{x}>1\right\}\right)=0$.

The lower bound for the increasing part of the spectrum uses P2 $\left(\mu, \rho,\left\{\left(x_{n}, \lambda_{n}\right)\right\}, h\right)$.

Proposition 4.10. Assume that $h_{c}(\mu)>0$ and that P2 $\left(\mu, \rho,\left\{\left(x_{n}, \lambda_{n}\right)\right\}, h_{c}(\mu)\right)$ holds. Then, for every $h \in\left[0, h_{c}(\mu)\right], d_{\nu_{\rho}}(h) \geq h$.

Proof. Let $h \in\left(0, h_{c}(\mu)\right]$ and let $\xi_{h}=\frac{d(1-\rho)+\rho h_{c}(\mu)}{h}$.

Condition P2 $\left(\mu, \rho,\left\{\left(x_{n}, \lambda_{n}\right)\right\}, h\right)$ yields that for some convenient choice of $\psi$ and $\left(\xi_{n}\right)_{n \geq 0}$ (converging to $\xi$ ), $d\left(\tau_{\mu}^{*}\left(h_{c}(\mu)\right), \rho, \xi\right)$ is a lower bound for the Hausdorff dimension of the set $T$ defined in $(2 \cdot 7)$. In this case, $d\left(\tau_{\mu}^{*}\left(h_{c}(\mu)\right), \rho, \xi\right)=h$. In addition, there exists a positive measure $m_{\rho, \xi}$ such that $m_{\rho, \xi}(T)>0$ and $m_{\rho, \xi}(E)=0$ for every set $E$ such that $\operatorname{dim} E<h$. Moreover, by Theorem $3 \cdot 2(\mathrm{i})$,

$$
E_{h}^{\nu \rho} \supset F_{h, \rho} \backslash\left(\bigcup_{h^{\prime}<h} G_{h^{\prime}, \rho}\right)=F_{h, \rho} \backslash \bigcup_{i \geq\left[h^{-1}\right]+1} G_{h-1 / i, \rho} .
$$

We remark that $T \subset F_{h, \rho}$. Indeed, every point of $T$ satisfies the property $\mathcal{P}\left(\rho, h_{c}(\mu), \xi_{h}, \varepsilon\right)$ for all $\varepsilon>0$ small enough.

The conclusion follows as in Proposition 4.9: Combining the estimates obtained in the proof of Proposition 4.7, we obtain $\operatorname{dim} F_{h-1 / i, \rho}<h$ for every $i \geq\left[h^{-1}\right]+1$. Moreover, part (iii) of Proposition 4.4 yields $\operatorname{dim}\left\{x \in \Omega: h_{\mu}(x) \leq h-1 / i\right\} \leq \tau_{\mu}^{*}\left(h-\frac{1}{i}\right)<h$. Thus $m_{\rho, \xi}\left(\bigcup_{i \geq\left[h^{-1}\right]+1} G_{h-1 / i, \rho}\right)=0$, which yields that $m_{\rho, \xi}\left(E_{h}^{\nu_{\rho}}\right) \geq m_{\rho, \xi}(T)>0$. This finally implies $\operatorname{dim} E_{h}^{\nu_{\rho}} \geq h$.

Notice that the points $x_{n}$ belong to $E_{0}^{\nu_{\rho}}$.

Proposition 4.11. Suppose that $\tau_{\mu}^{\prime}\left(0^{+}\right)>d$. Let $h \in\left[h_{c}(\mu), h_{\rho}(\mu)\right)$. Assume that either (P2 $\left(\mu, \rho,\left\{\left(x_{n}, \lambda_{n}\right)\right\}, \alpha(h)\right)$ holds) or $(\rho=1$ and $\mathbf{P 3}(\mu, h)$ holds $)$.

Then $d_{\nu_{\rho}}(h) \geq \tau_{\mu}^{*}(\alpha(h))$.

Proof. Recall Theorem 3·2(i). In the case where $h=h_{c}(\mu)$ or $\rho=1$, when $\mathbf{P 3}(\mu, h)$ holds, the proof is the same as that of Proposition $4 \cdot 9$, since $\alpha(h)=h$ and $\left(\widetilde{E}_{h}^{\mu} \cap\{x\right.$ : $\left.\left.\xi_{x}=1\right\}\right) \subset E_{h, \rho}$.

We suppose that $\rho<1$ and that $\mathbf{P 2}\left(\mu, \rho,\left\{\left(x_{n}, \lambda_{n}\right)\right\}, \alpha(h)\right)$ holds. We proceed as in the 
The multifractal nature of heterogeneous sums of Dirac masses

proof of Proposition 4·10. Let $\xi_{h}=\frac{d(1-\rho)+\rho \tau_{\mu}^{*}(\alpha(h))}{\tau_{\mu}^{*}(\alpha(h))}$. As above, P2 $\left(\mu, \rho,\left\{\left(x_{n}, \lambda_{n}\right)\right\}, h\right) \mathrm{im}-$ plies that for some convenient choice of $\psi$ and $\left(\xi_{n}\right)_{n \geq 0}$ converging to $\xi, d\left(\tau_{\mu}^{*}(\alpha(h)), \rho, \xi_{h}\right)=$ $\tau_{\mu}^{*}(\alpha(h))$ is a lower bound for the Hausdorff dimension of the limsup-set $T(2 \cdot 7)$. Moreover, there is a positive measure $m_{\rho, \xi_{h}}$ such that $m_{\rho, \xi_{h}}(T)>0$ and for every set $E$ with $\operatorname{dim} E<\tau_{\mu}^{*}(\alpha(h)), m_{\rho, \xi_{h}}(E)=0$. Finally, $T \subset F_{h, \rho}$ because every point of $T$ satisfies $\mathcal{P}\left(\rho, \alpha(h), \xi_{h}, \varepsilon\right)$ for all $\varepsilon>0$.

Since $h \mapsto \tau_{\mu}^{*}(\alpha(h))$ is increasing on $\left(h_{c}(\mu), h_{\rho}(\mu)\right)$ and $\tau_{\mu}^{*}(h)<\tau_{\mu}^{*}(\alpha(h))$ (Proposition 4.8) the arguments used in the proof of Proposition 4.10 yield $\operatorname{dim} E_{h}^{\nu_{\rho}} \geq \tau_{\mu}^{*}(\alpha(h))$.

\section{Examples}

In [11], several suitable systems and statistically self-similar measures illustrate the notion of heterogeneous ubiquity. Some of them are recalled here.

\section{5·1. Some suitable systems}

- The family of the b-adic numbers:

Fix $b$ an integer $\geq 2$. The family $\left\{\left(x_{n}, \lambda_{n}\right)\right\}_{n}=\left\{\left(\mathbf{k} b^{-j}, b^{-j}\right)\right\}_{j \geq 0, k \in\left\{0, \ldots, b^{j}-1\right\}^{d}}$ obviously has all the appropriate properties.

- The family of the rational numbers:

The system $\left\{\left(\mathbf{p} / q, 2 / q^{1+1 / d}\right)\right\}$, where $q \in \mathbb{N}^{*}, \mathbf{p}=\left(p_{1}, p_{2}, \ldots, p_{d}\right) \in\{0, \ldots, q-1\}^{d}$ such that at least one fraction $p_{i} / q$ is irreducible, is weakly redundant and satisfies $\mathbf{P} 4$.

- The family of the $\{\{n \alpha\}, 1 / n\}_{n \in \mathbb{N}}$ :

Let $\alpha$ be an irrational number, such that its approximation degree by the family of rational numbers above in the one dimensional case equals 1 . The family $\{(\{n \alpha\}, 1 / n)\}_{n \geq 1}$ is weakly redundant and satisfies $\mathbf{P} 4(\{x\}$ stands for the fractional part of $x)$. Weak redundancy does not hold if the approximation degree of $\alpha$ is $>1$.

- Poisson point processes.

Let $S$ be a Poisson point process with intensity $\ell \otimes \pi$ in the square $[0,1] \times(0,1]$, where $\ell$ denotes Lebesgue measure on $[0,1]$ and $\pi$ is a positive locally finite Borel measure on $(0,1]$ (see $[\mathbf{2 4}]$ for the construction of a Poisson point process). Write this set $S$ as $\left\{\left(y_{n}, r_{n}\right)\right\}_{n}$. Let $\beta=\inf \left\{\gamma: \int_{x \leq 1} x^{\gamma} \pi(d x)<\infty\right\}$. There exists a non-decreasing sequence $\beta_{n}$ converging to $\beta$ such that the system $\left\{\left(y_{n}, r_{n}^{\beta_{n}}\right)\right\}_{n}$ is weakly redundant and satisfies $\mathbf{P} 4$ (see [11] for more details).

- Random family based on uniformly distributed points:

Let $\left\{x_{n}\right\}_{n}$ be a sequence of points independently and uniformly distributed in $[0,1]^{d}$ and $\left\{\lambda_{n}\right\}_{n}$ a non-increasing sequence of positive numbers.

If $\lim \sup _{n \rightarrow+\infty}\left(\sum_{p=1}^{n} \lambda_{p} / 2-d \log n\right)=+\infty$ and $\lim \sup _{j \rightarrow \infty} j^{-1} \log _{2} \# T_{j}=1\left(T_{j}\right.$ was defined in (1.5)), then the system $\left\{\left(x_{n}, \lambda_{n}\right)\right\}_{n}$ satisfies $\mathbf{P} 4$.

\subsection{Random self-similar measures satisfying conditions $\mathbf{P} \mathbf{1 - 3}$ for suitable systems}

We mention two important classes of such measures. The first one is obtained through the thermodynamic formalism. The second one contains limits of $[0,1]^{d}$-martingales considered in the multiplicative chaos in the meaning of $[\mathbf{2 1}]$. It is shown in $[\mathbf{9}, \mathbf{1 0}, \mathbf{1 1}]$ that these measures $\mu$ obey conditions P1-3 for suitable systems $\left\{\left(x_{n}, \lambda_{n}\right)\right\}_{n}$ (including those of Section 5.1) when $h$ ranges in the interval where $\tau_{\mu}^{*}(h)>0$. 
- (Random) Gibbs measures.

These measures are obtained as fixed points of adjoints of Ruelle-Perron Fröbenius operators associated with a Hölder potential $\phi$ in the dynamical system $\left([0,1)^{d}, T\right)$, where $T(x)=c x \bmod 1$ with $c \in \mathbb{N} \backslash\{0,1\}$ - see $[\mathbf{3 3}]$-. Random counterparts of these measures are considered in $[\mathbf{2 2}, \mathbf{1 7}, \mathbf{9}]$.

- $[0,1]^{d}$-martingales.

The first examples are the independent multiplicative cascades, or Mandelbrot martingales introduced in $[\mathbf{2 7}]$ and then studied extensively in $[\mathbf{2 7}, \mathbf{2 3}, \mathbf{1 8}, \mathbf{2 9}, \mathbf{2 8}, \mathbf{1}, \mathbf{1 5}$, $\mathbf{4}, \mathbf{5}, \mathbf{1 0}]$. They are a particular case of a wider class of $[0,1]^{d}$-martingales - see $[\mathbf{7}]-$ which satisfy condition $\mathbf{P 2}\left(\mu, \rho,\left\{\left(x_{n}, \lambda_{n}\right)\right\}, h\right)$. This class also includes compound Poisson cascades introduced in $[\mathbf{6}]$, as well as their extension in $[\mathbf{3}]$, and other examples (see $[\mathbf{7}, \mathbf{8}, \mathbf{1 0}]$ for details).

Notice that we are able to prove that $\mathbf{P} \mathbf{2}\left(\mu, \rho,\left\{\left(x_{n}, \lambda_{n}\right)\right\}, h\right)$ holds true for these measures only in the case $\rho=1$ (see [10] for details).

- The substitute to independent multiplicative cascade in the critical case of degeneracy.

These measures are constructed in [4] (using results of [26]) in order to study the end points of the multifractal spectrum of independent multiplicative cascades. A modification of the martingale used in the independent multiplicative cascades definition is involved in their construction. In our context, the interesting property of these measures is that they provide examples of measures $\mu$ such that $q_{c}(\mu)=1$ but $h_{c}(\mu)=0$ - see [10] - which satisfies condition $\mathbf{P 2}\left(\mu, \rho,\left\{\left(x_{n}, \lambda_{n}\right)\right\}, h\right)$ when $\rho=1$.

\section{Comments}

When $\rho=1$ and conditions P1-3 are satisfied for every $h \in \operatorname{supp}\left(d_{\mu}\right)$, the multifractal spectrum of $\nu_{1}$ (associated with a suitable weakly redundant system) becomes the Legendre transform of the function $\widetilde{\tau_{\nu_{1}}}$ defined by

$$
\widetilde{\tau_{\nu_{1}}}: q \mapsto\left\{\begin{array}{ll}
\tau_{\mu}(q) & \text { if } q \leq q_{c}(\mu) \\
0 & \text { if } q>q_{c}(\mu)
\end{array} .\right.
$$

The same lines of computation as in [8] apply here (up to a small correction due to the weak redundancy assumption). As a result, if $\nu_{1}$ is the measure associated with this system and with a suitable measure $\mu$, then $\tau_{\nu_{1}}=\widetilde{\tau_{\nu_{1}}}$. This implies that the Hausdorff multifractal spectrum of $\nu_{1}$ is the Legendre spectrum of its scaling function $\tau_{\nu_{1}}$. Consequently, $\nu_{1}$ fulfills the (box [13] or centered [30]) multifractal formalisms for measures.

The context is quite different when $\rho<1$, and the computations are subtler. In further work, we will see that if $\mu$ is non trivial, all the usual multifractal formalisms fail on the right of $h_{c}(\mu)$ in this case.

\section{REFERENCES}

[1] M. Arbeiter and N. Patzschke, Random self-similar multifractals, Math. Nachr., 181 (1996), 5-42.

[2] V. Aversa and C. BAndt, The multifractal spectrum of discrete measures, Acta Univ. Car., 31(21) (1990), 5-8.

[3] E. BACRY and J.-F. MuZY, Log-infinitely divisible multifractal processes, Commun. Math. Phys. 236 (2003), 449-475. 
[4] J. BARral, Continuity of the multifractal spectrum of random statistically self-similar measures, J. Theor. Probab. 13 (2000), 1027-1060.

[5] J. BARral and F. Ben NASR and J. Peyrière, Comparing multifractal formalisms: the neighboring condition, Asian J. Math. 7 (2003), 149-166.

[6] J. Barral and B. Mandelbrot, Multifractal products of cylindrical pulses, Prob. Theory Relat. Fields 124 (2002), 409-430.

[7] J. BARRAl and B. MANDELBrot, Random multiplicative multifractal measures, in Fractal Geometry and Applications: A Jubilee of Benoît Mandelbrot, Proc. Symp. Pure Math., 72, Part 2, pp 3-90, AMS, Providence, RI (2004).

[8] J. Barral and S. Seuret, Combining multifractal additive and multiplicative chaos, Commun. Math. Phys. 257 (2005), 473-497.

[9] J. Barral and S. Seuret, Inside singularity sets of random Gibbs measures. J. Stat. Phys. 120(5-6), 1101-1124, 2005.

[10] J. BARral and S. SEuret, Renewal of singularity sets of statistically self-similar measures. Preprint (2004). ArXiv math.PR/0503421.

[11] J. Barral and S. Seuret, Heterogeneous ubiquitous systems in $\mathbb{R}^{d}$ and Hausdorff dimension, Preprint (2004). ArXiv math.GM/0503419

[12] J. Bertoin, Lévy processes, Cambridge University Press (1996).

[13] G. Brown and G. Michon and J. Peyrière, On the multifractal analysis of measures, J. Stat. Phys. 66 (1992), 775-790.

[14] M.M. Dodson and M.V. Melián and D. Pestana and S.L. Vélani, Patterson measure and Ubiquity, Ann. Acad. Sci. Fenn. Ser. A I Math. 20, 37-60 (1995).

15] K.J. FALCONER, The multifractal spectrum of statistically self-similar measures, J. Theor. Prob. 7 (1994), 681-702.

[16] K.J. Falconer, Representation of families of sets by measures, dimension spectra and Diophantine approximation, Math. Proc. Camb. Phil. Soc. 128 (2000), 111-121.

[17] A.H. FAn, Multifractal analysis of infinite products, J. Stat. Phys. 86 (1997), 5/6:13131336.

[18] R. Holley and E.C. Waymire, Multifractal dimensions and scaling exponents for strongly bounded random fractals, Ann. Appl. Probab. 2 (1992), 819-845.

[19] S. JAFFARD, Old friends revisited: The multifractal nature of some classical functions, $J$. Fourier Anal. Appl. 3 (1997), 1-22.

[20] S. Jaffard, The multifractal nature of Lévy processes, Probab. Theory Relat. Fields, 114 (1999), 207-227.

[21] J.-P. Kahane, Positive martingales and random measures, Chi. Ann. of Math 8B1 (1987), $1-12$.

[22] Y. KifER, Fractals via random iterated function systems and random geometric constructions, Fractal geometry and stochastics (Finsterbergen, 1994), Progr. Probab. 37, 145164, Birkhäuser, Basel (1995).

[23] J.-P. Kahane and J. Peyrière, Sur certaines martingales de Benoît Mandelbrot, Adv. in Math. 22 (1976), 131-145.

[24] J.F.C. Kingman, Completely random measures, Pacific J. Math. 21 (1967), 59-78.

[25] J. LÉvy VÉHel and R. VoJak, Multifractal analysis of Choquet capacities, Adv. Appl. Math. 20 (1998), 1-43.

[26] Q. Liu, On generalized multiplicative cascades, Stoch. Proc. Appl. 86 (2000), 263-286.

[27] B.M. Mandelbrot, Intermittent turbulence in self-similar cascades: divergence of high moments and dimension of the carrier, J. Fluid Mech. 62 (1974), 331-358.

[28] G.M. Molchan, Scaling exponents and multifractal dimensions for independent random cascades, Commun. Math. Phys. 179 (1996), 681-702.

[29] L. OlsEn, Random geometrically graph directed self-similar multifractals, Pitman Research Notes in Mathematics Series, 307, 1994.

[30] L. Olsen, A multifractal formalism, Adv. Math. (1995) 116, 92-195.

[31] L. Olsen, Self-affine multifractal Sierpinski sponges in $\mathbb{R}^{d}$, Pacific J. Math., 183 (1998).

[32] Y. Pesin, H. Weiss, The multifractal analysis of Gibbs measures: motivation, mathematical foundation, and examples, Chaos 7(1), 89-106 (1997)

[33] W. PARry and M. Policott, Zeta functions and the periodic orbit structure of hyperbolic dynamics, Société Mathématique de France, Astérisque (1990), 187-188. 